ACCEPTED MANUSCRIPT

\title{
A hierarchical method for the impact force reconstruction in composites structures
}

To cite this article before publication: Mario Emanuele De simone et al 2018 Smart Mater. Struct. in press https://doi.org/10.1088/1361$665 \mathrm{X} / \mathrm{aae} 11 \mathrm{c}$

\author{
Manuscript version: Accepted Manuscript \\ Accepted Manuscript is "the version of the article accepted for publication including all changes made as a result of the peer review process, \\ and which may also include the addition to the article by IOP Publishing of a header, an article ID, a cover sheet and/or an 'Accepted \\ Manuscript' watermark, but excluding any other editing, typesetting or other changes made by IOP Publishing and/or its licensors" \\ This Accepted Manuscript is @ 2018 IOP Publishing Ltd.
}

During the embargo period (the 12 month period from the publication of the Version of Record of this article), the Accepted Manuscript is fully protected by copyright and cannot be reused or reposted elsewhere.

As the Version of Record of this article is going to be / has been published on a subscription basis, this Accepted Manuscript is available for reuse under a CC BY-NC-ND 3.0 licence after the 12 month embargo period.

After the embargo period, everyone is permitted to use copy and redistribute this article for non-commercial purposes only, provided that they adhere to all the terms of the licence https://creativecommons.org/licences/by-nc-nd/3.0

Although reasonable endeavours have been taken to obtain all necessary permissions from third parties to include their copyrighted content within this article, their full citation and copyright line may not be present in this Accepted Manuscript version. Before using any content from this article, please refer to the Version of Record on IOPscience once published for full citation and copyright details, as permissions will likely be required. All third party content is fully copyright protected, unless specifically stated otherwise in the figure caption in the Version of Record.

View the article online for updates and enhancements. 


\title{
A Hierarchical Method for the Impact Force Reconstruction in Composites
}

\author{
Structures \\ Mario Emanuele DE SIMONE, Francesco CIAMPA, Michele MEO* \\ Mechanical Engineering Department, University of Bath, Bath, United Kingdom \\ *email:m.meo@bath.ac.uk
}

Key words: Impact force reconstruction, Time-reversal, Radial Basis Functions, Composite Materials

\section{ABSTRACT}

Impact damage is a major concern for new generation aircraft composite components due to their low impact resistance capabilities. The development of an impact location and force reconstruction algorithm would provide rapid and efficient prediction of damage occurrence, thus making structures safer and creating maintenance inspection procedures more efficient, thus saving time and costs. However, state-of-the-art impact force reconstruction algorithms use reference data from numerical simulations and require a detailed knowledge of mechanical properties, which are difficult to obtain under real operational conditions.

This paper presents a hierarchical impact force reconstruction algorithm that relies on experimental structural responses measured by a sparse array of surface bonded receiving ultrasonic transducers. This algorithm uses time reversal method to retrieve the location of an impact source and interpolation techniques based on hierarchical radial basis functions to calculate the transfer function at the impact point and reconstruct the impact force history. A number of impact testing were performed on a composite plate-like structure and a wing stringer-skin panel, and compared with impact force algorithms available in literature. Experimental results revealed that the proposed impact force reconstruction method was able to extrapolate the information associated with points far from the impact location and determine the impact force history with high level of accuracy in a real aircraft structure. Since the proposed algorithm requires the calibration of transfer functions from a very 
sparse training set of data and it does not need numerical models of the component under investigation, it demonstrates its potential as a useful monitoring tool for impact force reconstruction in composite components for full-scale aircraft structural applications leading to timely and costefficient inspections.

\section{INTRODUCTION}

Composite materials exhibit desirable physical and chemical properties that include lightweight coupled with high stiffness and strength and, in the last few decades, have been widely used in many industrial sectors, from aerospace to civil and nuclear. However, composite structures may experience significant material degradation if impacted by low-velocity objects such as tool drops, runway debris and hail stones. Hence, in order to prevent serious and dangerous consequences due to micro-cracks and barely visible impact damage (BVID), a number of structural health monitoring (SHM) techniques capable of localising the impact source and reconstructing the force history (or energy) in composites have been investigated [1-3]. These SHM methods enhance the efficiency of current material inspection systems and enable the prediction of damage severity. The so-called "inverse approach" has been widely used for the determination of the force history in different materials and components. In this approach, the impact energy can be detected through the knowledge of structural responses acquired by a set of transducers surface bonded to the specimen [4]. However, the inverse approach leads to a deconvolution for numerical solutions, which is a well-known ill-posed mathematical problem that can be numerically unsolvable or, if the solution exists, can be instable for the presence of small disturbance such as noise. To overcome this issue, Doyle solved the inverse problem by using the fast Fourier transform method in both isotropic [5] and orthotropic [6] plates. However this method needed cumbersome windowing and filtering processes in order to suppress the effect of wave reflection from the plate boundaries. Chang and Sun [7] proposed a method suitable for composite structures based on the generation of the experimental Green's function using time signal deconvolutions. However, this technique was limited by (i) a scaling factor for the 
identification of the absolute amplitude of the Green's function and (ii) the short duration of responses that were characterized by a low signal-to-noise ratio. Wu et al. [8] utilised an optimisation method to reconstruct the time history of the impact force on a circular plate, which was subject to impact loading at its centre. In this work, Green's functions were generate from a series of Bessel functions. The authors also proposed an experimental method based on a deconvolution process, which unfortunately did not provide accurate results when simultaneous impacts acted at multiple locations. The accuracy in reconstructing the impact force was further enhanced by developing an analytical method suitable for composite laminates [9], which was based on the classical lamination theory and the Rayleigh-Ritz equations. A detailed theoretical explanation of the deconvolution problem for impact force reconstruction and the regularisation methods for its solution was also presented by Jacquelin et al. [4]. Nevertheless, this approach required the determination of unknown regularisation parameters, as well as a-priori knowledge of mechanical properties, which is often difficult to obtain. Despite this limitation, several authors proposed algorithms based on regularisation techniques. Kalhori et al. $[10,11]$ proposed some research works based on $l_{2}$-norm-based regularisation methods (Tikhonov regularisation), whilst Qiao et al. [12,13] and Pan et al. [14] presented methodologies based on $l_{1}$-norm regularisation. Moreover Yan et al. [15] developed a two-loops algorithm based on the Bayesian interference regularisation and a nonlinear unscented Kalman filter (UKF), both applied to a state-space dynamic model of a composite plate under impact. Park et al. [16] introduced an inverse method based on a system identification technique able to establish a model constructed with transfer functions. This method is suitable for complex structures using a distributed sensor array. Chen et al. [17,18] and Xu et al. [19] proposed a similar approach by using four transducers fixed on both isotropic and composite plates. A completely different approach for impact force reconstruction is based on the artificial neural network (ANN) method (see [20-24]), which consists of complex mathematical models that can be trained with scattered data. However, due to the large amount of training data to be generated, the ANN approach is considered impractical for SHM applications. 
The aim of this research work was to develop an impact force reconstruction method that relies on the structural responses measured by three surface bonded receiving sensors. The proposed algorithm was divided into two steps. In the first one, the impact localisation was achieved by using the time reversal (TR) method [25-28]. In the second step, an interpolation technique based on the hierarchical radial basis function $(\mathrm{RBF})$ approach was used to calculate the transfer function at the impact location and reconstruct the impact force history [36]. Radial basis functions were here used as they are able to approximate functions or data with high level of accuracy, which are only available at sparse locations $[37,39,40]$. Moreover one of the greatest advantages of this approach is related to its versatility in multi-dimensional applications. For their peculiar characteristics, radial basis functions are used in many fields including numerical finite element methods for the solution of partial differential equations [46], neural networks and machine learning [48,51], statistical approximation [53], geophysical research [47], ultrasonic imaging [55] and engineering applications such as the analysis of orthotropic shells and boundary condition reconstruction on an elastic cavity [52]. In order to validate the proposed hierarchical impact force reconstruction algorithm, a number of experimental tests were performed on a composite plate and a wing stringer-skin panel. Moreover, further comparison with a method available in literature, namely the shape function (SF) interpolation technique [16], was performed. The outline of this research work is as follows: in Section 2, the impact localisation algorithm based on the time reversal method is described. The impact force reconstruction algorithm is presented in Section 3, whose main parts are the Section 3.1, where a suitable transfer function calculation method is presented, and the Section 3.2, whose topic is the interpolation trough the radial basis functions. Section 4 shows the set-up used to perform experimental tests, whilst in Section 5 all the results are illustrated. The conclusions of this paper are presented in Section 6. 


\section{IMPACT LOCALISATION - TIME REVERSAL METHOD}

A number of impact localisation algorithms were developed for both isotropic and anisotropic media without requiring a-priori knowledge of the mechanical properties of the material [29-32]. In this work, the impact source localisation was the initial stage of the impact force reconstruction algorithm that, in turn, allowed reconstructing the impact force magnitude through information available in points close to the impact event. The TR method was here used for the impact localisation. TR is based on the hypothesis of time invariance and spatial reciprocity of elastodynamic wave equation and on the Huygens' principle, through which it is possible to reconstruct the wave function in a generic volume by the knowledge of its sources located on a two-dimensional surface. A detailed theoretical explanation of TR method is presented in [25-27]. TR method/is typically split into the "forward propagation" and the "backward propagation" steps. In the "forward" one, low-velocity impacts were applied in $M$ excitation points, also called "calibration points", which were arbitrarily chosen on the plane of the structure (focusing plane) that identifies the monitoring zone. A number of $N$ receiving sensors were used, so that a set of $N \times M$ signals was acquired and stored in the computer memory. These waveforms represent the response of the structure (e.g. displacements, velocities, strains, etc.) subject to impact loading. With the hypothesis of free unbounded space and assuming that the wave field, $u(\boldsymbol{r}, t)$, can be measured at any point of a closed surface $S$, the general solution of the elastodynamic wave equation is:

$$
u(\boldsymbol{r}, t)=\iiint_{\Omega}\left[G\left(\boldsymbol{r}, t ; \boldsymbol{r}_{0}\right) \otimes p\left(\boldsymbol{r}_{0}, t\right)\right] d \Omega_{0},
$$

where $\otimes$ represents a convolution over time, $p\left(\boldsymbol{r}_{0}, t\right)$ indicates an impulsive force located in $\boldsymbol{r}_{0}$ and $G\left(\boldsymbol{r}, t ; \boldsymbol{r}_{0}\right)$ is the Green space-time function. If the excitation function is a Dirac delta function (unit impulse function), the Green function is called "impulse response" and it is equal to the measured wave field.

As aforementioned, the surface of the structure was divided into a discrete domain composed of $M$ excitation points, so that Eq. (1) can be re-written as: 


$$
u(\boldsymbol{r}, t)=\sum_{m=1}^{M} G\left(\boldsymbol{r}, t ; \boldsymbol{r}_{m}\right) \otimes p\left(\boldsymbol{r}_{m}, t\right)=\sum_{m=1}^{M}\left[\int_{0}^{t} G\left(\boldsymbol{r}, t-\tau ; \boldsymbol{r}_{m}\right) p\left(\boldsymbol{r}_{m}, \tau\right) d \tau\right]
$$

where $\tau$ is the time lag. It should be noted that only if the structure deformation are considered linearly elastic and small enough to neglect geometric nonlinearities, the relationship between the impact force and the response of the structure can be considered as linear and mathematically described by the linear convolution reported in Eq. (2). The "backward propagation step" consisted of correlating the waveform emitted by a point of unknown position $\boldsymbol{r}_{m 0}$ with all the impulse responses stored in the "forward propagation" step. It can be demonstrated (see for instance [25-27]) that the impact location is calculated as the maximum of the following time reversal operator (i.e. when $\boldsymbol{r}_{m}=\boldsymbol{r}_{m 0}$ ):

$$
R_{\mathrm{TR}}=G\left(\boldsymbol{r}_{m}, t ; \boldsymbol{r}\right) \otimes G\left(\boldsymbol{r},-t ; \boldsymbol{r}_{m 0}\right)=\int_{0}^{t} G\left(\boldsymbol{r}_{m}, t ; \boldsymbol{r}\right) G\left(\boldsymbol{r}, t+\tau ; \boldsymbol{r}_{m 0}\right) d \tau,
$$

which represents a cross correlation operation. The Cauchy-Schwarz inequality proves that [33]:

$$
\begin{array}{r}
\left|R_{\mathrm{TR}}\right|^{2}=\left|\int_{0}^{t} G\left(\boldsymbol{r}_{m}, t ; \boldsymbol{r}\right) G\left(\boldsymbol{r}, t+\tau ; \boldsymbol{r}_{m 0}\right) d \tau\right|^{2} \\
\quad \leq \int_{0}^{t}\left|G\left(\boldsymbol{r}_{m}, t ; \boldsymbol{r}\right)\right|^{2} d \tau \int_{0}^{t}\left|G\left(\boldsymbol{r}, t+\tau ; \boldsymbol{r}_{m 0}\right)\right|^{2} d \tau,
\end{array}
$$

where $|\cdot|$ is the absolute value. $\mathrm{Eq}(4)$ is equivalent to:

$$
\left|R_{\mathrm{TR}}\right| \leq\left(\int_{0}^{t}\left|G\left(\boldsymbol{r}_{m}, t ; \boldsymbol{r}\right)\right|^{2} d \tau\right)^{\frac{1}{2}}\left(\int_{0}^{t}\left|G\left(\boldsymbol{r}, t+\tau ; \boldsymbol{r}_{m 0}\right)\right|^{2} d \tau\right)^{\frac{1}{2}}
$$

The Euclidean norm is defined as:

$$
\left\|G\left(\boldsymbol{r}_{m}, t ; \boldsymbol{r}\right)\right\|=\left(\int_{0}^{t}\left|G\left(\boldsymbol{r}_{m}, t ; \boldsymbol{r}\right)\right|^{2} d t\right)^{\frac{1}{2}}
$$

The signal energy is defined as:

$$
E_{G \boldsymbol{r}_{m}}=\int_{0}^{t}\left|G\left(\boldsymbol{r}_{m}, t ; \boldsymbol{r}\right)\right|^{2} d t
$$

Comparing Eqs. (6) and (7), Eq. (5) becomes: 


$$
\left|R_{\mathrm{TR}}\right| \leq\left\|G\left(\boldsymbol{r}_{m}, t ; \boldsymbol{r}\right)\right\|\left\|G\left(\boldsymbol{r}, t+\tau ; \boldsymbol{r}_{m 0}\right)\right\|=\sqrt{E_{G \boldsymbol{r}_{m}} E_{G \boldsymbol{r}_{m 0}}}
$$

As a measure of similarity of two signals, the correlation coefficient was used, which is defined as:

$$
c_{\mathrm{TR}}=\max \left(\frac{\left|R_{\mathrm{TR}}\right|}{\sqrt{E_{G \boldsymbol{r}_{m}} E_{G \boldsymbol{r}_{m 0}}}}\right) .
$$

The expression (9) satisfies the inequality $0 \leq c_{\mathrm{TR}} \leq 1$. The $c_{\mathrm{TR}}$ coefficient is close to one when the signals are similar (i.e. at the true impact location), whilst it is close to zero elsewhere. In order to compensate the incoherent measurement noise due to electronics, an average from the contribution of the $N$ receiving sensors was here used and a single mean correlation coefficient was related to each grid node. According to Figure 1, each cell of the grid on the monitoring zone is identified by four nodes and it was possible to perform a further mean among the correlation coefficients associated to each node in order to calculate a global correlation coefficient of the cell, indicated as $c_{\mathrm{TR} \_\mathrm{GLOBAL}}$.

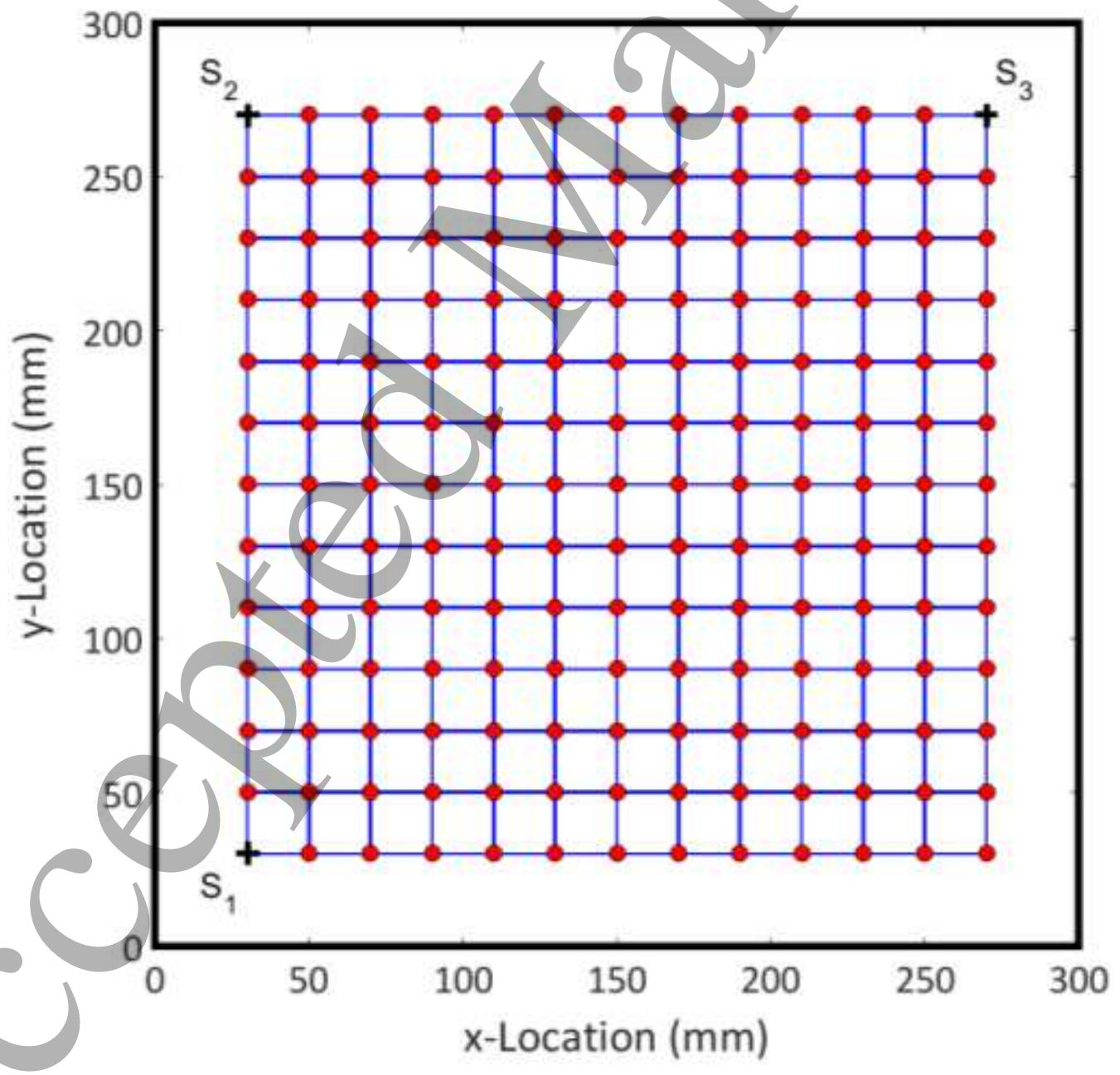

Figure 1. Initial surface grid. Calibration points are depicted as red spots. 
The cell where $c_{\text {TR_GLOBAL }}$ was maximum was regarded as the cell including the unknown impact point and here named as the "impact cell". The described TR approach is depicted in Figure 2. The impact coordinates were estimated by a centre-of-gravity method, in a similar way to $[18,19]$ :

$$
x_{I}=\frac{\sum_{i=1}^{4} x_{i} c_{\mathrm{TR}_{i}}}{\sum_{i=1}^{4} c_{\mathrm{TR}_{i}}}, \quad y_{I}=\frac{\sum_{i=1}^{4} y_{i} c_{\mathrm{TR}_{i}}}{\sum_{i=1}^{4} c_{\mathrm{TR}_{i}}},
$$

where $x_{i}$ and $y_{i}$ are the coordinates of the $i^{\text {th }}$ node of the impact cell, $c_{\mathrm{TR}_{i}}$ is the averaged correlation coefficient related to the $i^{\text {th }}$ node, $x_{I}$ and $y_{I}$ are the estimated locations of the current impact event.

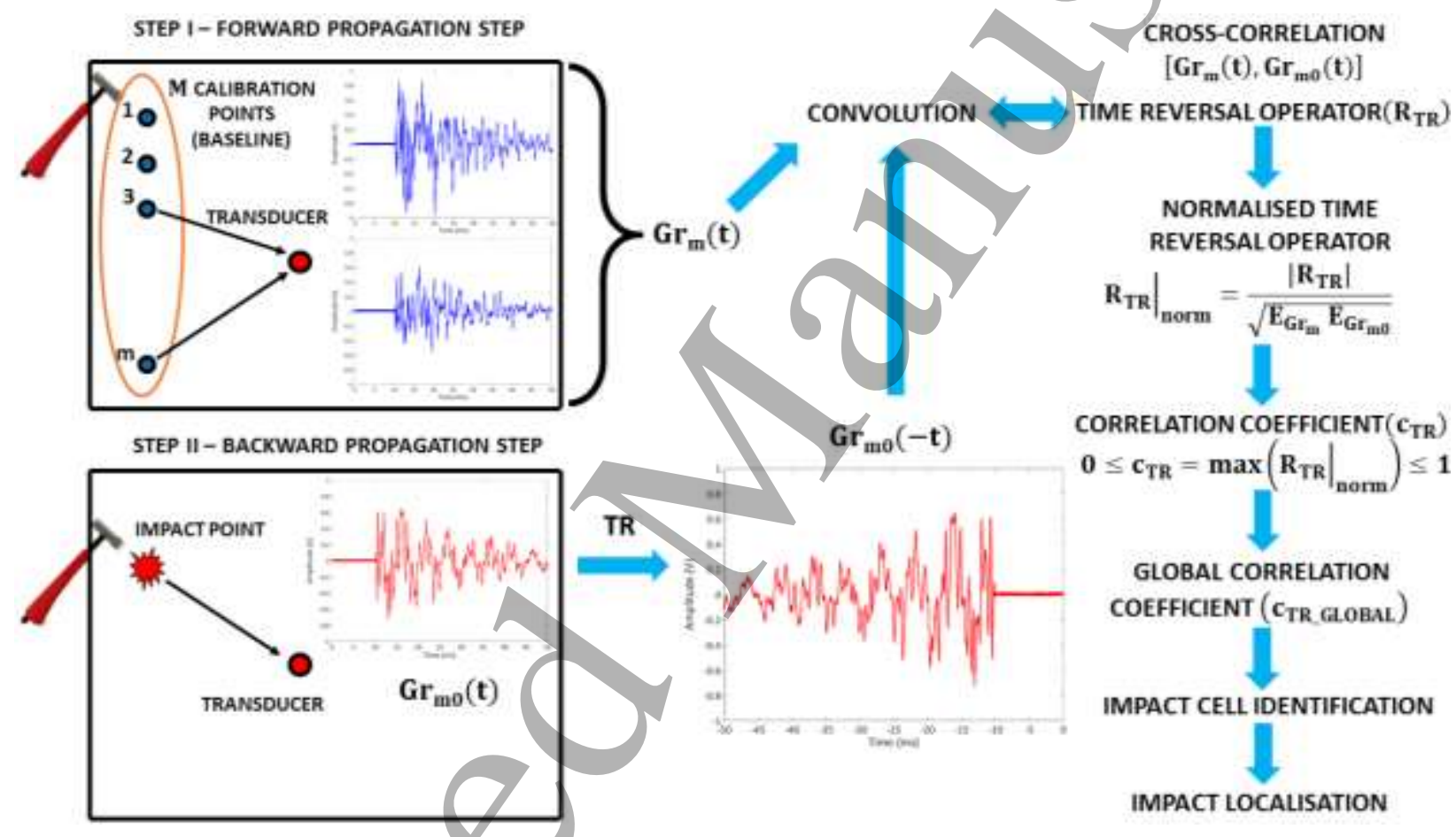

Figure 2. Graphical illustration of both "forward" and "backward" steps in the time reversal process for impact localisation.

\section{IMPACT FORCE RECONSTRUCTION}

For the impact force reconstruction, an impact force $p(t)$ acting on the surface of a specimen and the structural response $u(t)$ acquired by a transducer fixed to the specimen were considered. From a signal processing perspective, Eq. (1) mathematically represents a linear convolution between two arbitrary signals, so it can be rewritten as: 


$$
u(t)=(G \otimes p)(t)=\int_{0}^{t} G(t-\tau) p(\tau) d \tau
$$

The aim of this research work was to recover the impact force from Eq. (11). As described in [4], a discrete problem must be solved by transforming the convolution integral [Eq. (10)] into a system of algebraic equations expressed in the following matrix form:

$$
[u]=[G][p],
$$

where $[u]$ is the response vector $n \times 1,[p]$ is the force vector of dimensions $n \times 1$ and $[G]$ is the transfer matrix of dimensions $n \times n$, where $n$ is the number of samples of acquired signals. The recovering of impact forces in Eq. (11) identifies an "inverse problem", known as "deconvolution problem", which is a well-known ill-posed system of equations with the $[G]$ matrix ill-conditioned. In order to overcome the difficulties related to a deconvolution problem in time domain, the frequency response function (FRF) of the impulsive structural response was considered. Indeed, according to the convolution theorem [34], the convolution of two time signals corresponds to a simple product of their spectra in the Fourier domain:

$$
u(t)=(G \otimes p)(t)=\int_{0}^{t} G(t-\tau) p(\tau) d \tau \Rightarrow U(f)=H(f) \cdot P(f)
$$

where $H(f)$ is the Fourier transform of the Green space-time function $G(t)$, indicated as the transfer function in the next sections. By following the TR process for impact localisation described in Section 2, the impact force reconstruction algorithm was divided into the following three steps:

1. the calculation of transfer function at each calibration point;

2. the hierarchical interpolation of transfer functions associated with a sparse array of points close to the identified impact location;

3. the impact force identification by using the estimated transfer function at impact location. The description of these three steps is provided in the following sub-sections. 


\subsection{Transfer function calculation}

An experimental method for the calculation of transfer function at each calibration point was here employed to prevent the use of approximated analytical and numerical models of the sample under investigation, which may poorly reconstruct the transfer function of the structure. This is the common case of components with complex geometries or when material properties are not available [35]. The identification of the transfer function is based on the cross-correlation function between the acquired response and the impact force, which is expressed by:

$$
S_{u p}(f)=U(f) \cdot P^{*}(f)
$$

By inserting Eq. (11) into Eq. (13), yields:

$$
S_{u p}(f)=H(f) \cdot P(f) \cdot P^{*}(f) .
$$

The auto-spectrum of the impact force is defined as:

$$
S_{p p}(f)=P(f) \cdot P^{*}(f)=|P(f)|^{2}
$$

therefore, Eq. (15) becomes:

$$
S_{u p}(f)=H(f) \cdot S_{p p}(f)
$$

and the transfer function, component by component, is calculated as reported below:

$$
H\left(f_{i}\right)=\frac{S_{u p}\left(f_{i}\right)}{S_{p p}\left(f_{i}\right)}
$$

Other studies consider the transfer function as the ratio between the auto-spectra of response and impact force, which represents only the modulus of the transfer function that does not contain any information on the phase of the system [35]. Conversely, the described approach provides both module and phase of transfer function. If nonlinear effects would not be negligible, the transfer functions associated to impacts with different energies could be calculated and the resulting average would be regarded as the transfer function of the system. However, this case was not investigated in the presented research work. At the end of the process a number of $N$ transfer functions are available at each calibration point. Figure 3 shows an example of transfer function calculation from one of the calibration points. 

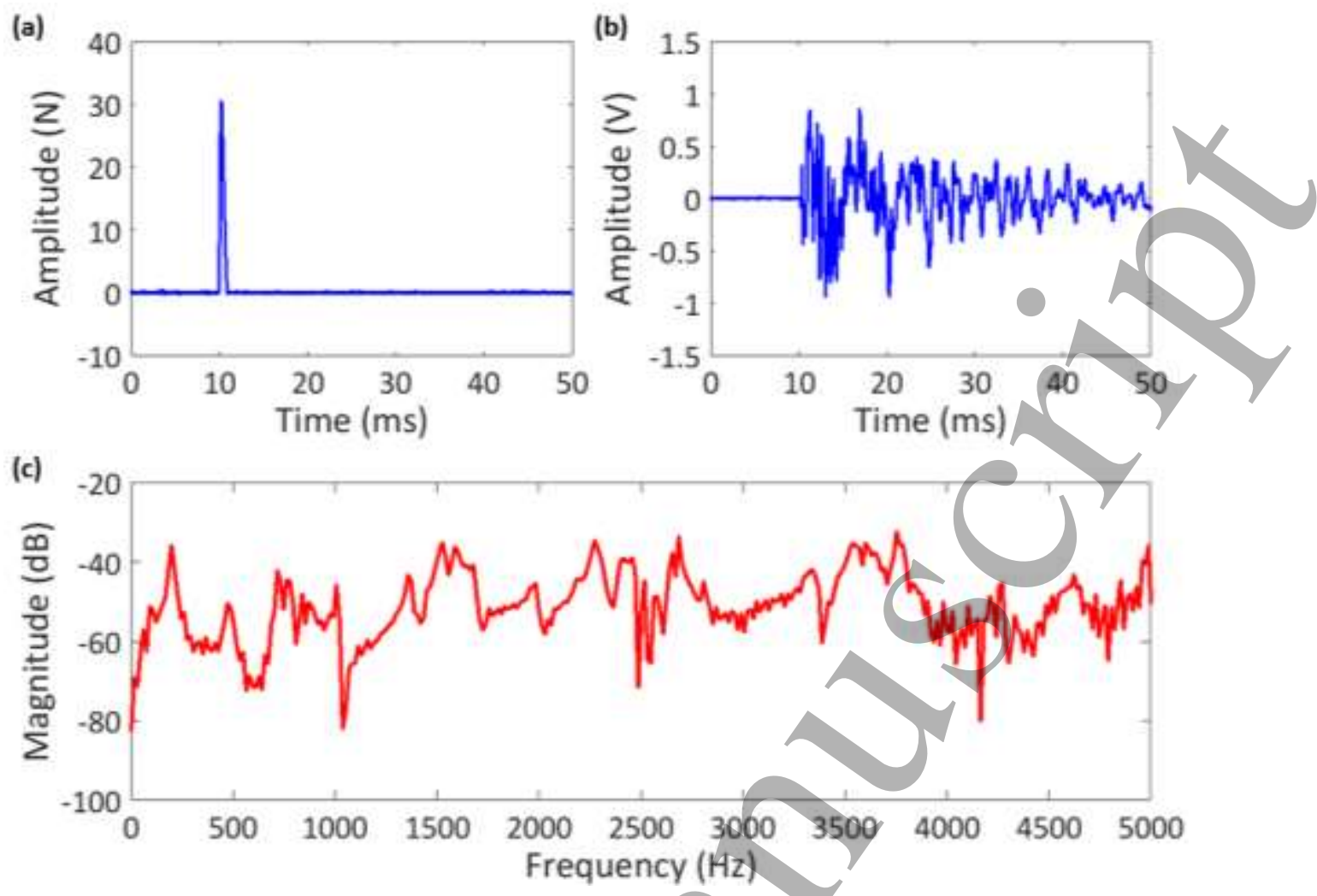

Figure 3. Transfer function calculation by using a single transducer: a) impact force; b) acquired response; c) calculated transfer function in the frequency domain.

\subsection{Radial basis function 2D interpolation}

As described in Section 2, it was possible to identify the impact location using the TR method [Eq. (11)]. The transfer function at the impact location can then be obtained by using an interpolation of transfer functions associated with the cell whose vertices are the four calibration points surrounding the impact location. In this regard, Park et al. firstly proposed the shape functions (SF) method, which uses polynomial interpolating functions (also known as shape functions) to reconstruct the impact force ([16-19]). The main idea of the SF technique is to relate the cell with a regular element of the same topology, whose edges have a non-dimensional length (see Figure 4). The corresponding nodes in this parametric space have coordinates $(\xi, \eta)$ and the transfer function at impact location in frequency domain can be expressed by:

$$
\bar{H}_{\left(x_{I}, y_{I}\right)}=\sum_{i=1}^{4} \lambda_{i} H_{i},
$$


where $H_{i}$ is the transfer function at $i^{\text {th }}$ point of the physical cell and $\lambda_{i}$ are the basis functions linking the physical and parametric coordinates. For two-dimensional bilinear interpolation, these functions are given by [16]:

$$
\begin{gathered}
\lambda_{1}(\xi, \eta)=(1-\xi)(1-\eta) \\
\lambda_{2}(\xi, \eta)=\xi(1-\eta) \\
\lambda_{3}(\xi, \eta)=(1-\xi) \eta \\
\lambda_{4}(\xi, \eta)=\xi \eta
\end{gathered}
$$

where it is $\xi=\frac{x-x_{1}}{x_{2}-x_{1}}$ and $\eta=\frac{y-y_{1}}{y_{3}-y_{1}}$.

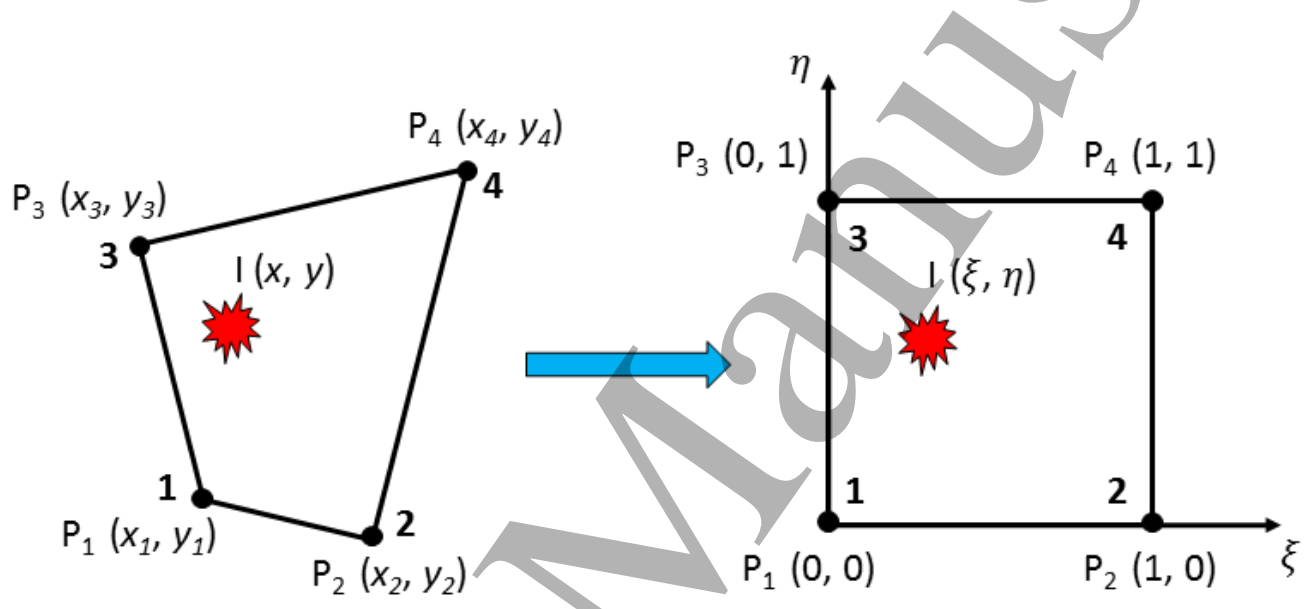

Figure 4. Illustration of the shape function (SF) interpolation method.

The SF method needs information related to four points close to the impact event. However, the higher is the distance from the impact event, the larger would the interpolation error.

To overcome this issue, in this paper a different interpolation method was used, which is based on the hierarchical radial basis functions (RBF) approach, analysed and developed in several research works. A detailed theoretical explanation of the RBF approach was presented by Wright [49]. The general idea of the RBF method is that for a given set of $n$ data points $\left\{x_{j}\right\}_{j=1}^{n}$ and corresponding data values $\left\{f_{j}\right\}_{j=1}^{n}$, a set of basis functions $\left\{\psi_{j}(x)\right\}_{j=1}^{n}$ is chosen such that a linear combination of these functions satisfies the interpolation conditions. For a one-dimensional function $s(x)$ it is possible to write: 


$$
s(x)=\sum_{j=1}^{n} \lambda_{j} \psi_{j}(x)
$$

where $\lambda_{j}$ are the expansion coefficients, determined by solving a linear system of equations based on the interpolation conditions $s\left(x_{j}\right)=f_{j}$ for $j=1, \ldots, n$. Many types of basis functions ensure this system is non-singular when the data points $\left\{x_{j}\right\}_{j=1}^{n}$ are distinct. In the case of one-dimensional data, a number of techniques such as the polynomial and Fourier interpolation are able to solve the described problem [56]. Conversely, these interpolation techniques are not suitable if data in more than one dimension are considered, so that the described approach is doomed to fail because the linear system of equations for determining the expansion coefficients becomes singular (according to the Haar's theorem [36]). This non-singularity problem can be overcome by creating an interpolating function approach that uses a linear combination of translates of a single basis function radially symmetric about its centre. This approach is referred to as the RBF method. This is a generalized version of the multiquadric (MQ) method, developed previously by Hardy [38] and then by Carlson [44] and Foley [45], for solving problems of topographic surface reconstruction from a set of sparse and scattered measurements from some source points. The general form of MQ interpolant in $d$ dimensions is expressed by:

$$
s(\boldsymbol{x})=\sum_{j=1}^{n} \lambda_{j} \sqrt{c^{2}+\left\|\boldsymbol{x}-\boldsymbol{x}_{j}\right\|^{2}}, \quad \boldsymbol{x}, \boldsymbol{x}_{j} \in \mathbb{R}^{d},
$$

where $\|\cdot\|$ denotes the Euclidean norm and $c \neq 0$ is a constant, introduced by Hardy for circular hyperboloid basis functions. The expansions coefficients $\lambda_{j}$ are determined from the interpolation conditions $s\left(\boldsymbol{x}_{\boldsymbol{j}}\right)=f_{j}$ for $j=1, \ldots, n$. Micchelli demonstrated the method was unconditionally nonsingular, also when a number of other basis functions are used, that, because of the radial symmetry about their centre, were called "radial basis functions" [41]. The basic RBF method can be expressed by the following interpolant: 


$$
s(\boldsymbol{x})=\sum_{j=1}^{n} \lambda_{j} \phi\left(\left\|x-x_{j}\right\|\right), \quad \boldsymbol{x}, \boldsymbol{x}_{j} \in \mathbb{R}^{d}
$$

where $\phi(r), r \geq 0$, is some radial function. As described above, the expansions coefficients $\lambda_{j}$ are determined from the interpolation conditions $s\left(\boldsymbol{x}_{\boldsymbol{j}}\right)=f_{j}$ for $j=1, \ldots, n$, which leads to the following symmetric linear system:

$$
[A][\lambda]=[f],
$$

where the entries of $A$ matrix are given by $a_{j, k}=\phi\left(\left\|x_{j}-x_{k}\right\|\right)$. Many radial basis functions are present in literature; some common examples of $\phi(r)$ are reported in Table 1 (see [50]).

Table 1. Some common types of radial basis functions.

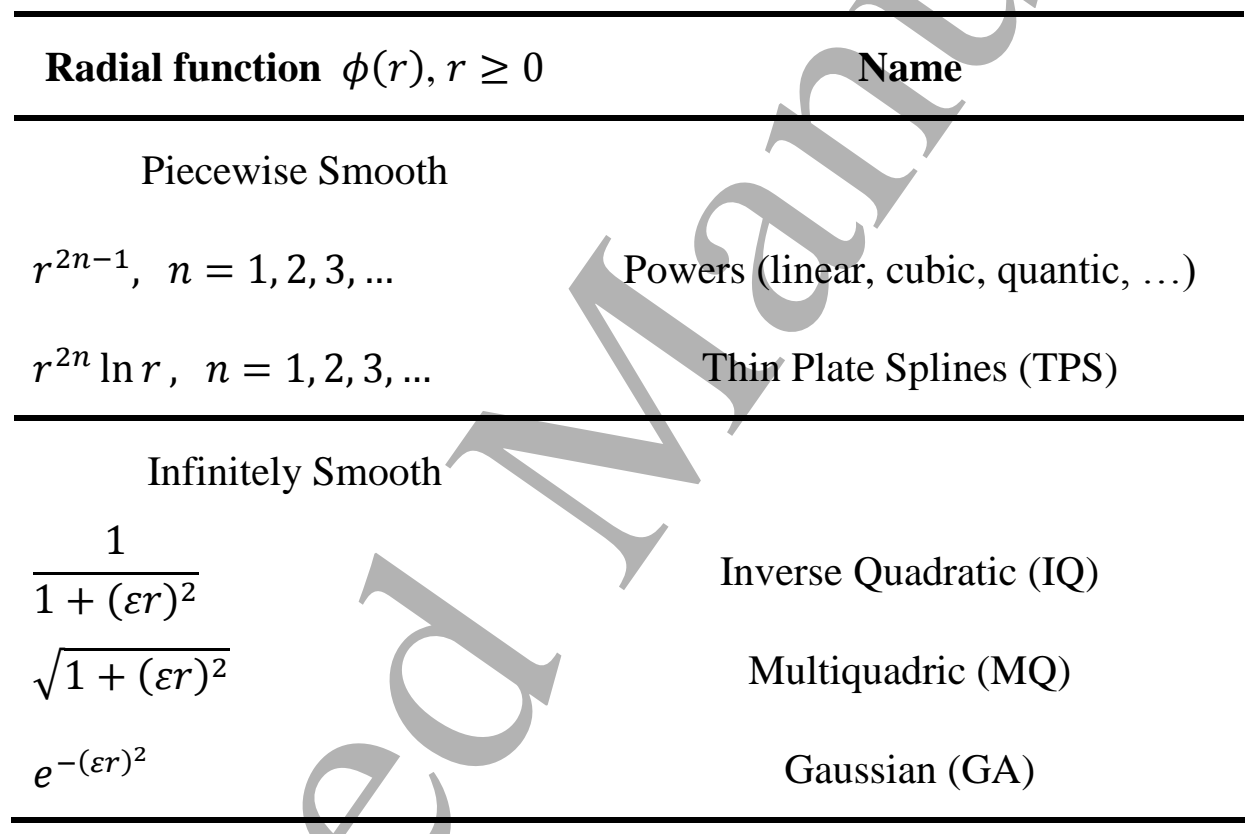

The thin plate spline (TPS) was used as radial basis function, whose kernel is $\varphi(r)=r^{2} \ln r$. This function was selected for its peculiar characteristics, as a smooth interpolation can be achieved with derivatives of any order and there are no free parameters requiring manual tuning. Furthermore there is a physical analogy involving the bending of a thin sheet of metal, because TPS is able to minimize the so-called "bending energy" [43]. Due to some stability issues, it is possible to consider additional polynomial terms to Eq. (23). The augmented RBF method can be expressed by the following interpolant: 


$$
s(\boldsymbol{x})=\sum_{j=1}^{n} \lambda_{j} \phi\left(\left\|\boldsymbol{x}-\boldsymbol{x}_{j}\right\|\right)+\sum_{k=1}^{L} \gamma_{k} p_{k}(\boldsymbol{x}), \quad \boldsymbol{x}, \boldsymbol{x}_{j} \in \mathbb{R}^{d}
$$

where $\left\{p_{k}(\boldsymbol{x})\right\}_{k=1}^{L}$ is a basis for $\prod_{l}\left(\mathbb{R}^{d}\right)$, that is the space of all $d$-variate polynomials that have degree less than or equal to $l$ and whose dimension is $L=\left(\begin{array}{c}d+l \\ d\end{array}\right)$. To account for the conditions from the additional polynomial terms, the following constraints are applied:

$$
\sum_{j=1}^{n} \lambda_{j} p_{k}\left(\boldsymbol{x}_{j}\right)=0, \quad k=1,2, \ldots, L
$$

The expansions coefficients $\lambda_{j}$ and $\gamma_{k}$ are determined from the interpolation conditions and the constraints (26), which lead to the following symmetric linear system:

$$
\left[\begin{array}{cc}
A & P \\
P^{T} & 0
\end{array}\right]\left[\begin{array}{l}
\lambda \\
\gamma
\end{array}\right]=\left[\begin{array}{l}
f \\
0
\end{array}\right]
$$

where $A$ is the $n \times n$ matrix presented in (24) and $P$ is the $n \times L$ matrix with entries $p_{k}\left(\boldsymbol{x}_{j}\right)$ for $j=$ $1, \ldots, n$ and $k=1, \ldots, L$. The Micchelli's theorem [42] admit that the augmented RBF method is uniquely solvable for the cubic and TPS RBFs when $l=1$ and the conditions on the data points $\left\{\boldsymbol{x}_{j}\right\}_{j=1}^{n}$ are satisfied. With $l=1$ and considering a two-dimensional approach $(d=2)$, a constant and linear polynomial is considered $(L=3)$, which leads the following RBF interpolant:

$$
s(x, y)=\sum_{j=1}^{n} \lambda_{j} \phi\left(\sqrt{\left(x-x_{j}\right)^{2}+\left(y-y_{j}\right)^{2}}\right)+\gamma_{o}+\gamma_{1} x+\gamma_{2} y,
$$

In this paper, RBF interpolant (28) is used for the transfer functions interpolation. As first step the expansion coefficients $\lambda_{j}$ and $\gamma_{k}$ are calculated by solving the linear system of equations (29), where the $f$ values are the known values of the transfer functions in an arbitrary set of calibration points. Then, they are used for the calculation of the unknown transfer function value at the impact location. This process needs to be performed for all samples of the transfer function. The matrix expression of the described approach is presented below [54]: 


$$
\left[\begin{array}{cccccc}
\phi_{1,1} & \ldots & \phi_{1, n} & x_{1} & y_{1} & 1 \\
\vdots & \ddots & \vdots & \vdots & \vdots & \vdots \\
\phi_{n, 1} & \ldots & \phi_{n, n} & x_{n} & y_{n} & 1 \\
x_{1} & \ldots & x_{n} & 0 & 0 & 0 \\
y_{1} & \ldots & y_{n} & 0 & 0 & 0 \\
1 & \ldots & 1 & 0 & 0 & 0
\end{array}\right]\left[\begin{array}{c}
\lambda_{1} \\
\vdots \\
\lambda_{n} \\
\gamma_{1} \\
\gamma_{2} \\
\gamma_{o}
\end{array}\right]=\left[\begin{array}{c}
f_{1} \\
\vdots \\
f_{n} \\
0 \\
0 \\
0
\end{array}\right]
$$

\subsection{Impact force identification}

The interpolation algorithm described in Section 3.2 was performed by considering the $N$ transfer functions available at each calibration point. Once obtained the $N$ transfer functions at impact location, it was possible to calculate the $N$ spectra of impact force through Eq. (13). The impact force in time domain was calculated by the inverse Fourier transform of the average impact spectrum as expressed by the following expression [19]:

$$
p(t)=\mathcal{F}^{-1}\left\{\frac{\left.P(f)\right|_{1}+\left.P(f)\right|_{2}+\left.P(f)\right|_{3}}{3}\right\}
$$

where $\left.P(f)\right|_{N}$ is the spectrum of the impact force calculated by using signals acquired by $N^{\text {th }}$ receiving sensor.

\section{EXPERIMENTAL SET-UP}

To validate the described algorithms, experimental impact tests were conducted on two specimens:

- a carbon fibre reinforced plastic (CFRP) plate with dimensions of $300 \times 300 \times 4 \mathrm{~mm}^{3}$ and lay-up sequence of $[45 /-45 / 90 / 0]_{2 S}$ (see Figure 5);

- an aluminium/CFRP composite wing stringer-skin panel provided by the courtesy of Airbus UK with dimensions of $3000 \times 1000 \times 4 \mathrm{~mm}^{3}$ (see Figure 6).

The impacts were generated by using a hand-held instrumented impact hammer (sensitivity factor $=$ $=2.215 \mathrm{mV} / \mathrm{N}$ ) connected to a signal conditioner both manufactured by Meggit-Endevco. Two different arrangements of three receiving sensors were chosen, in order to demonstrate the validity of the described approach independently from the transducer locations: 
- three surface-bonded piezoelectric transducers (PIC 255) with diameter of $6.5 \mathrm{~mm}$ and thickness of $0.3 \mathrm{~mm}$ were used and located at corners of the CFRP plate;

- three acoustic emission transducers with $150 \mathrm{kHz}$ central frequency provided by the courtesy of Airbus UK.

The Cartesian reference frame was chosen with the origin at the bottom left corner of both the CFRP plate and the wing panel, where the monitoring area consists of a grid arranged with equally spaced nodes, which are the calibration points of the proposed TR algorithm. The spacing between them is $20 \mathrm{~mm}$ in both samples under investigation. The signals were acquired using a fourchannel oscilloscope with 16 bits of resolution, a sampling rate of $2 \mathrm{MHz}$ and an acquisition window of $50 \mathrm{~ms}$. All algorithms were implemented by the authors by using a MATLAB software code. The experimental set-up is showed in Figure 5 and Figure 6.

(a)

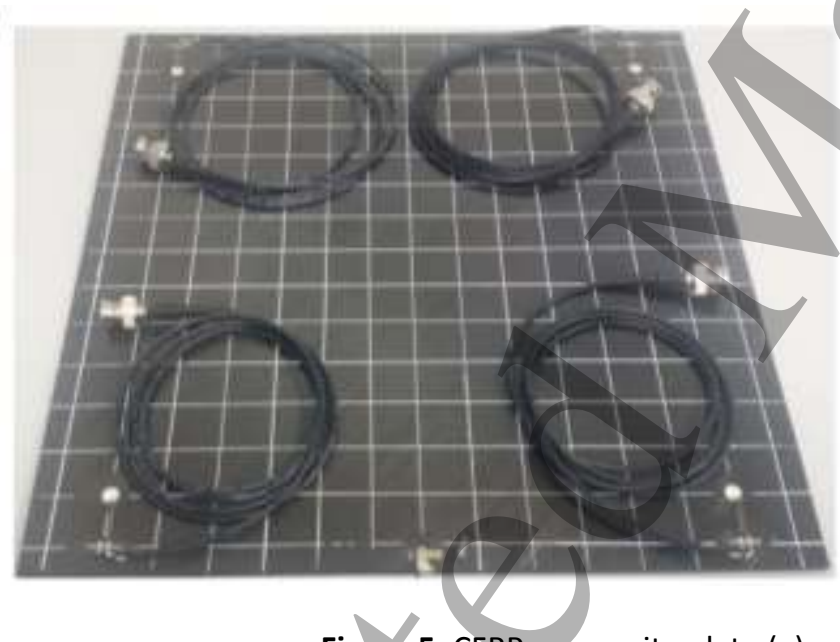

(b)

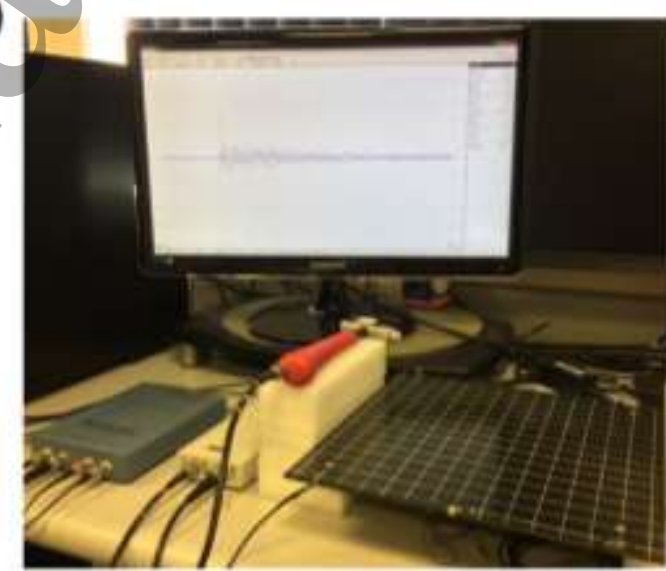

Figure 5. CFRP composite plate (a) and experimental set-up (b). 
(a)

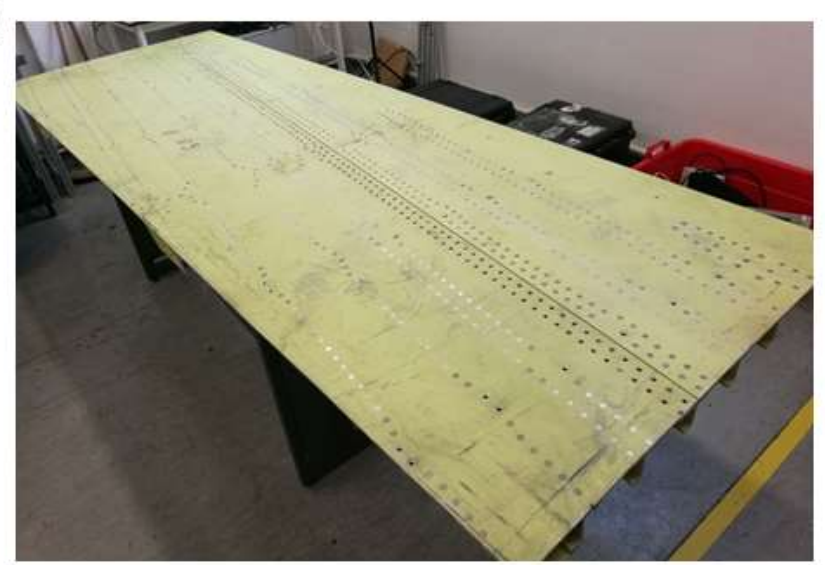

(b)

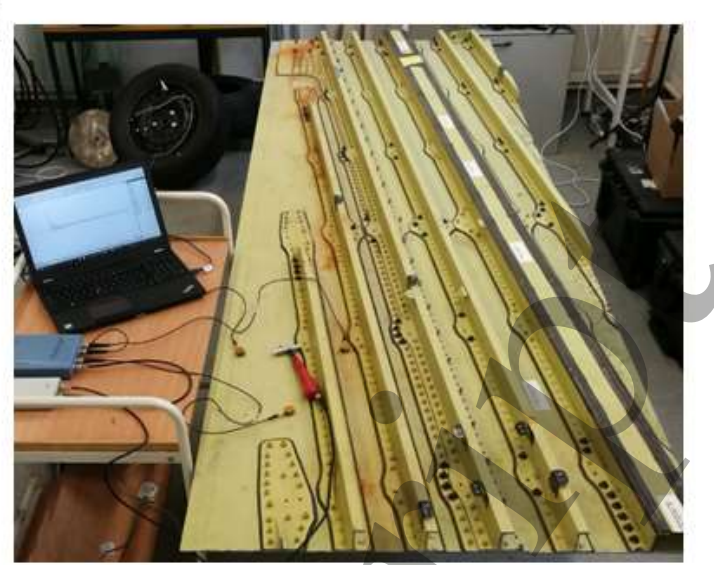

Figure 6. Wing stringer-skin panel: top view (a) and bottom view (b).

Table 2. Sensor coordinates on both specimens.

\begin{tabular}{lcccc}
\hline & \multicolumn{2}{c}{ x-Coordinate $(\mathrm{mm})$} & \multicolumn{1}{c}{-Coordinate $(\mathrm{mm})$} \\
& CFRP plate & Wing panel & CFRP plate & Wing panel \\
\hline Sensor 1 & 30 & 30 & 30 & 30 \\
Sensor 2 & 30 & 30 & 270 & 270 \\
Sensor 3 & 270 & 270 & 270 & 150 \\
\hline
\end{tabular}

\section{RESULTS}

The result section will be divided in three sub-sections: in Sec. 5.1 the impact localisation results on both specimens obtained with time reversal will be shown and discussed, whilst Secs 5.2, 5.3 and 5.4 report the impact force histories, calculated by using both the radial basis function and shape function interpolation methods. For these three sub-sections, three different sets of calibration points are considered:

- $\quad$ Set 1 , which consists of the four closest to impact location calibration points. These points represent the corners of an impact cell with dimension $20 \times 20 \mathrm{~mm}^{2}$ used for both interpolation methods;

- Set 2, which consists of four calibration points far from the impact location. These points represent the corners of an impact cell with dimension $60 \times 60 \mathrm{~mm}^{2}$, used for both interpolation methods; 
- $\underline{\text { Set } 3}$, in which we suppose that the information related to the four closest to impact location calibration points are not available. For the SF interpolation method, eight far points arranged in two impact cells are considered, whilst twelve points equally disposed in a square around the impact location are used for the RBF interpolation method.

The accuracy of the impact localisation method is expressed by the following formula for the location error $\Psi[57]$ :

$$
\Psi=\sqrt{\left(x_{\text {real }}-x_{\text {calculated }}\right)^{2}+\left(y_{\text {real }}-y_{\text {calculated }}\right)^{2}}
$$

where $\left(x_{\text {real }}, y_{\text {real }}\right)$ are the coordinates of the true impact position and $\left(x_{\text {calculated }}, y_{\text {calculated }}\right)$ are the coordinates of the impact location using the TR algorithm. Several methods can be also used to estimate the accuracy of the force reconstruction algorithms. In this paper, an error based on time integral of the force is considered that is given by the following equation [35]:

$$
\Gamma=\frac{\int_{t_{1}}^{t_{2}}\left|p_{\text {real }}(t)-p_{\text {reconstructed }}(t)\right| d t}{\int_{t_{1}}^{t_{2}} p_{\text {real }}(t) d t}
$$

where $T=t_{2}-t_{1}$ is an interval of the recording, which includes the impact force. By experimentally observing the time histories of measured data, two time intervals surrounding the impact peak were here used, i.e. $1 \mathrm{~ms}$ and $3 \mathrm{~ms}$.

It will be shown that the impact forces reconstructed by using the two different interpolation methods are exactly the same under the assumption that the same set of four interpolation points is used. As aforementioned in the Sec. 3.2, the RBF interpolation method overcomes the limitation of considering only four points and provides more accurate results by using the available information related to points far from the impact source. 


\subsection{Impact localisation results}

The initial calibration process at each grid point was performed, considering the same (constant) amplitude of the impact forces generated with the modal hammer. Therefore, information associated with impact force histories and structural responses acquired by the three receiving sensors were known. The three transfer functions at each grid point were calculated through the method described in Section 3.1. A set of impact tests were performed on the two specimens at arbitrary locations. Figure 7, Figure 8 and Table 3 show the real impact location and that calculated by the TR algorithm on both specimens (see Section 2). The four nodes of the impact cells depicted in Figure 7 and Figure 8 represent the set 1 and their coordinates are presented in Table 3.

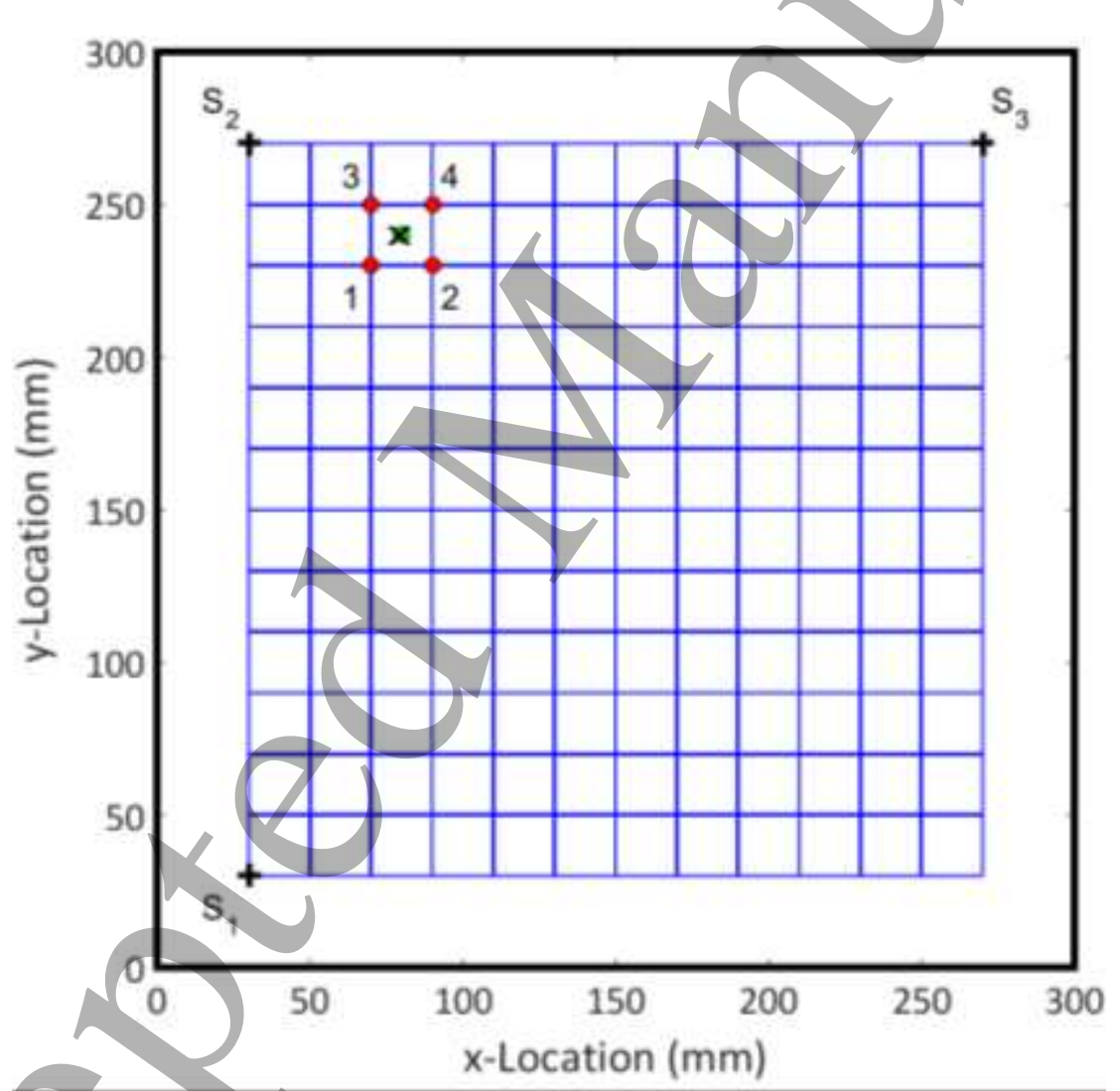

Figure 7. Source location on the CFRP plate by using time reversal method. The actual impact location is shown as a green circle, whilst the calculated one is shown as a cross $(x)$. The set 1 is shown with red circles. 


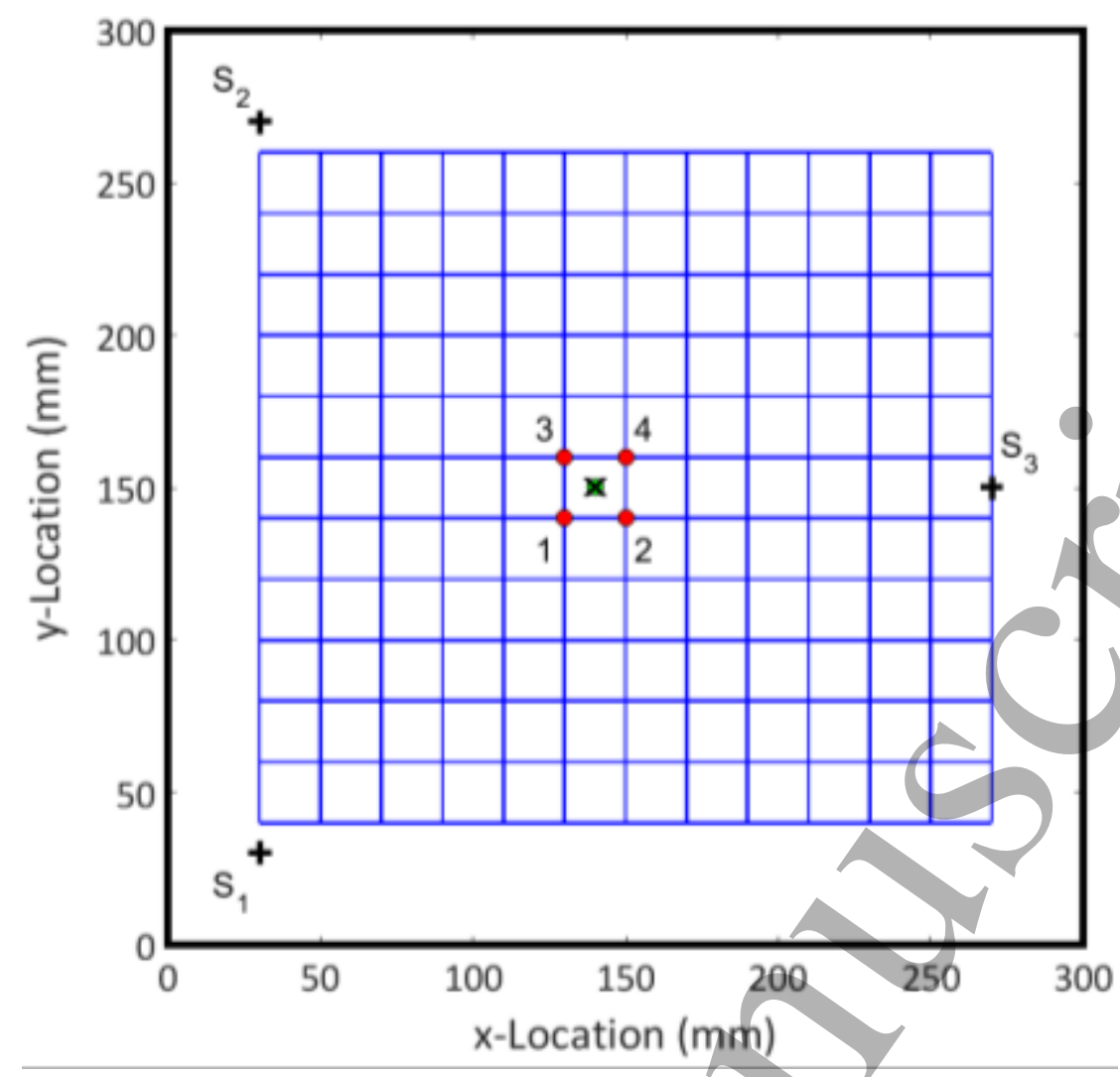

Figure 8. Source location on a portion of the wing panel by using time reversal method. The actual impact location is shown as a green circle, whilst the calculated one is shown as a cross $(X)$. The set 1 is shown with red circles.

Table 3. Coordinates of set 1 of interpolation points, impact positions and errors on both specimens.

\begin{tabular}{|c|c|c|c|c|}
\hline & x-Coor & te $(\mathrm{mm})$ & \multicolumn{2}{|c|}{$\mathrm{y}$-Coordinate (mm) } \\
\hline & CFRP pla & Wing panel & CFRP plate & Wing panel \\
\hline Node 1 & & 130 & 230 & 140 \\
\hline Node 2 & & 150 & 230 & 140 \\
\hline Node 3 & 70 & 130 & 250 & 160 \\
\hline No & 90 & 150 & 250 & 160 \\
\hline Current & 80 & 140 & 240 & 150 \\
\hline Calculated impact & 79.42 & 140.13 & 239.91 & 150.16 \\
\hline Location error $\Psi(\mathrm{mm})$ & \multicolumn{2}{|c|}{0.59} & \multicolumn{2}{|c|}{0.21} \\
\hline
\end{tabular}

Since the main purpose of this paper was to illustrate the performance of the proposed force reconstruction algorithm using hierarchical functions, only one impact location is reported in Figure 5 and Figure 6 for clarity reasons. In reality, more than fifty impacts were applied on both samples, showing great accuracy for all of them. However, by using a spacing of $20 \mathrm{~mm}$ between nodes and 
considering a high number of impact events (more than 50) at different positions along the plane of the specimens, location errors were always less than $1 \mathrm{~mm}$. For further information about the efficiency and accuracy of TR, please refer to other papers from the same group (e.g. [25-27]) and from other authors (e.g. [28]).

\subsection{Impact force reconstruction results - Set 1 of calibration points}

Figure 9 shows the transfer functions related to set 1 on the CFRP plate. Three transfer functions are available at each node, therefore the average transfer function associated with each node is presented in the following figure.

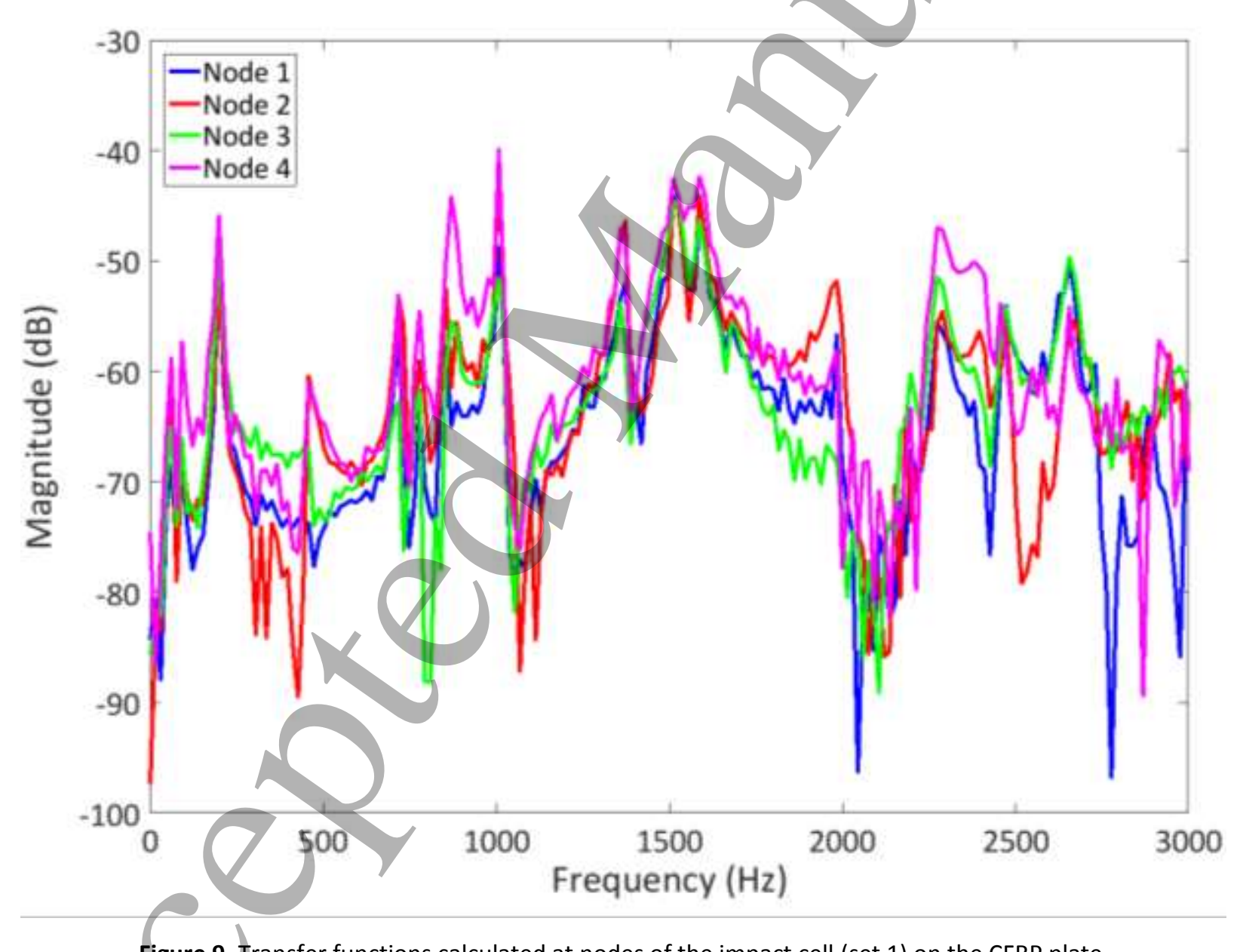

Figure 9. Transfer functions calculated at nodes of the impact cell (set 1) on the CFRP plate.

The interpolation process was performed three times since three sets of transfer functions were available at each node of the impact cell. Figure 10, related to the CFRP plate, depicts the comparison 
between the average transfer function at impact location calculated during the initial calibration process, and the interpolated ones obtained by using both the SF and the proposed RBF interpolation methods, averaged over the three receiving sensors.

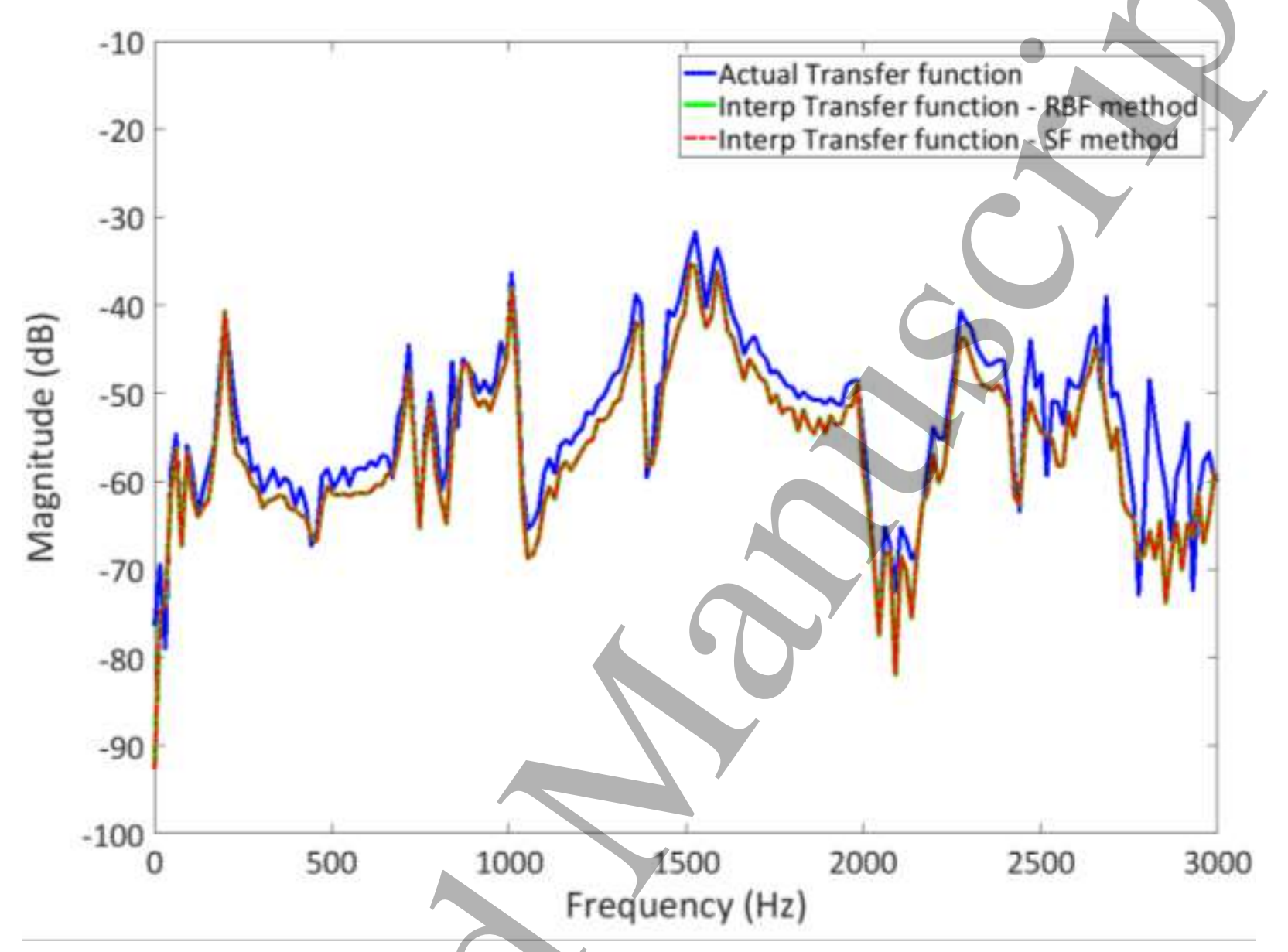

Figure 10. Comparison between the actual transfer function and the interpolated ones at the impact location on the CFRP plate.

As showed in Figure 10 the transfer functions calculated with the two interpolation methods are the same. Moreover, because of interpolation points are very close to the impact location, both interpolated results presented a negligible difference with the original transfer function. This assertion can be deducted also by considering Figure 11 and Figure 12, which present the comparison between the current impact force history and the reconstructed ones for the set 1 on both specimens and the signals acquired by all the receiving sensors (see Section 3.3). 


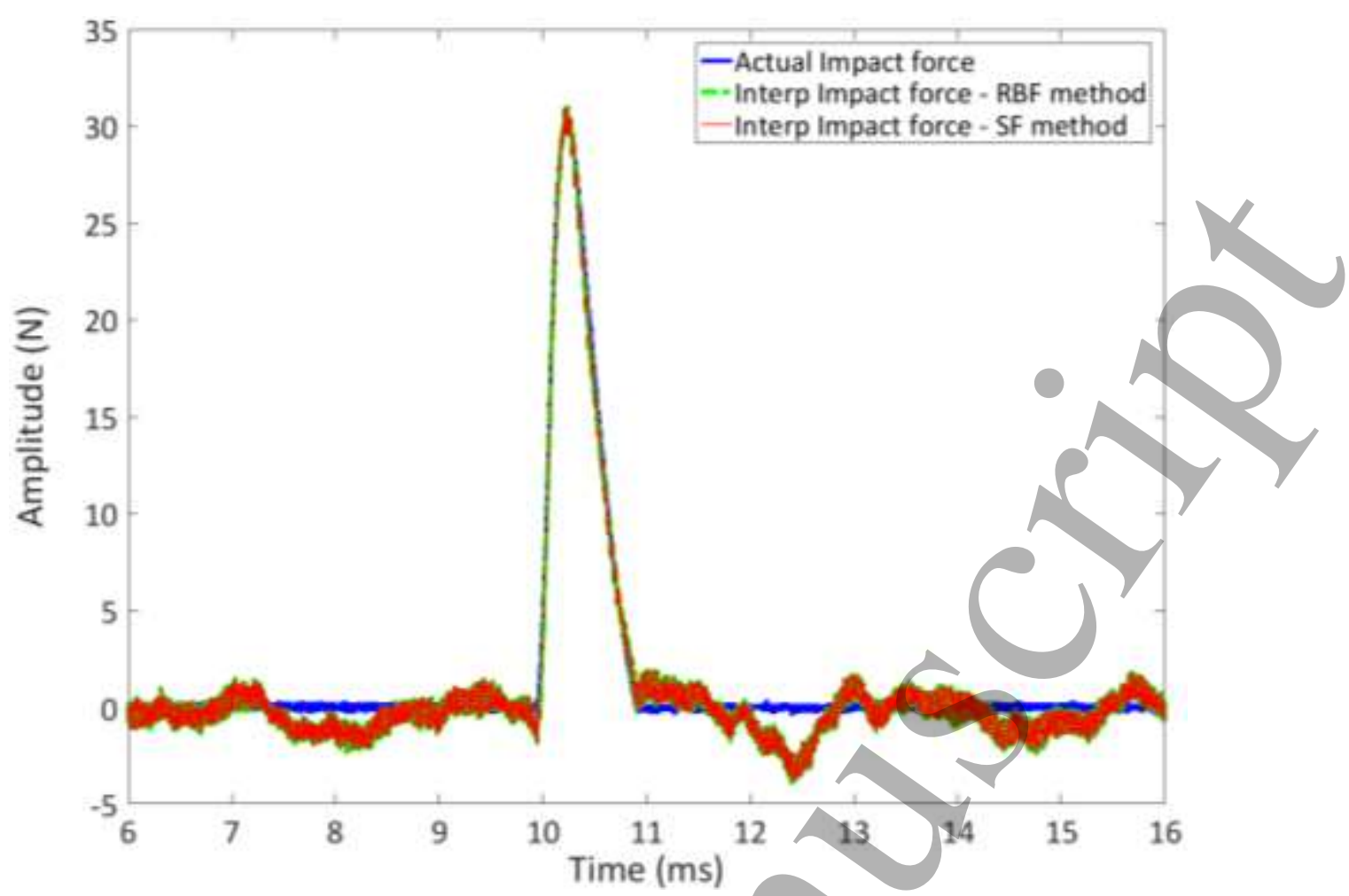

Figure 11. Comparison between the actual impact force and the interpolated ones by using radial basis function (RBF) and shape function (SF) methods. Set 1 of interpolation points on the CFRP plate is considered (see Figure 7). 
Dimensionless errors of both reconstructed impact forces with respect to the actual one on the two specimens are showed in Table 4. They are obtained by using Eq. (32).

Table 4. Comparison between impact forces considering set 1 of interpolation points on both specimens.

\begin{tabular}{cccccc}
\hline & \multicolumn{4}{c}{ INTERPOLATED IMPACT FORCES } \\
\hline & & \multicolumn{3}{c}{ RBF method } & \multicolumn{2}{c}{ SF method } \\
\hline \multirow{2}{*}{ Error $\Gamma$} & $T=1 \mathrm{~ms}$ & 0.05 & 0.1 & 0.05 & 0.1 \\
& $T=3 \mathrm{~ms}$ & 0.12 & 0.37 & 0.12 & 0.37 \\
\hline
\end{tabular}

As aforementioned, the two interpolation algorithms generated the same results with a small error with respect to the original signal, especially in the detection of impact peak amplitude. It should be noted that the error increases if the considered time window is wider, this because of the signal fluctuations due to the interpolation process.

\subsection{Impact force reconstruction results - Set 2 of calibration points}

Figure 14 and Table 6 depict the same comparison by now considering the set 2 of interpolation points on the two specimens, which are far from the impact location. The coordinates of the new set are reported in Table 5. In Figure 13 and Figure 14 the set 2 is shown with red circles, whilst the other calibration points are depicted with blue circles.

Table 5. Coordinates of set 2 of interpolation points on both specimens.

\begin{tabular}{|c|c|c|c|c|}
\hline & \multicolumn{2}{|c|}{$\mathrm{x}$-Coordinate $(\mathrm{mm})$} & \multicolumn{2}{|c|}{$\mathrm{y}$-Coordinate $(\mathrm{mm})$} \\
\hline & CFRP plate & Wing panel & CFRP plate & Wing panel \\
\hline Node 1 & 50 & 110 & 210 & 120 \\
\hline Node 2 & 110 & 170 & 210 & 120 \\
\hline Node 3 & 50 & 110 & 270 & 180 \\
\hline Node 4 & 110 & 170 & 270 & 180 \\
\hline
\end{tabular}


(a)

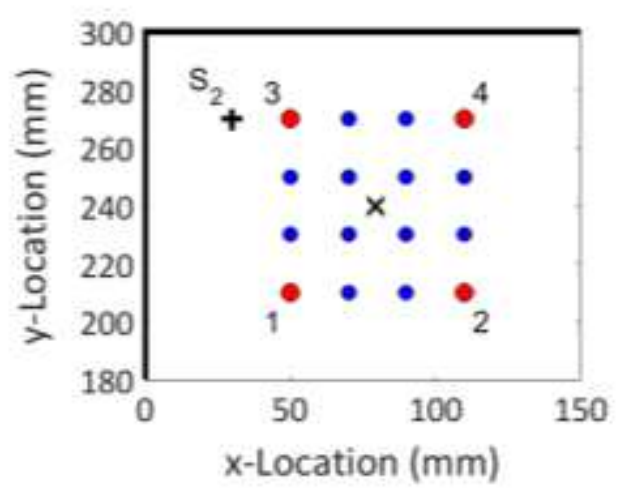

(b)

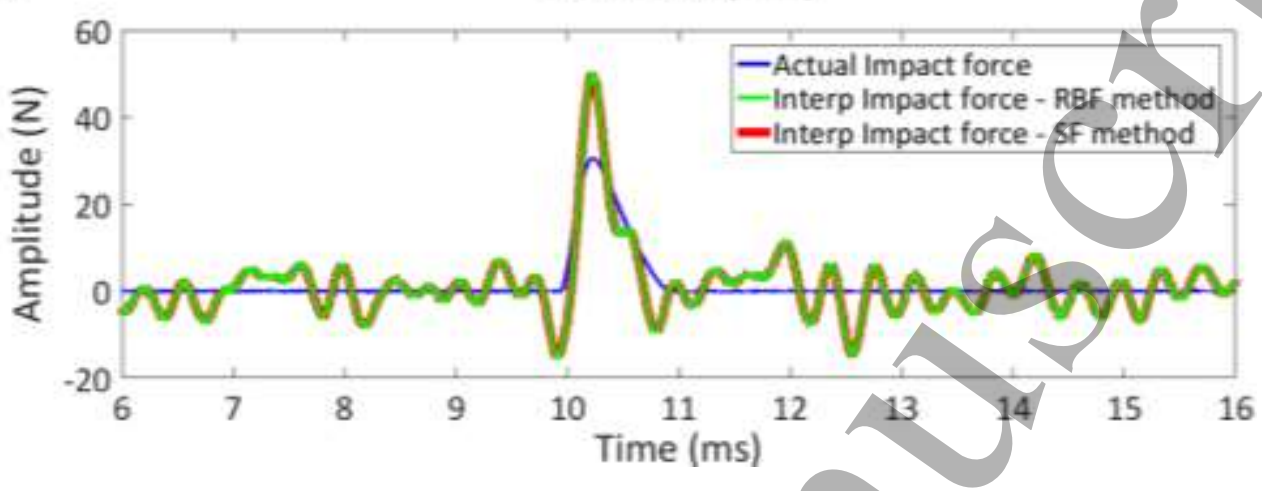

Figure 13. a) Zoom on the CFRP plate with a reduced section of the initial grid. The calculate impact location is shown as a cross $(X)$. b) Comparison between the actual impact force and the interpolated ones by using radial basis function (RBF) and shape function (SF) interpolation methods.

(a)

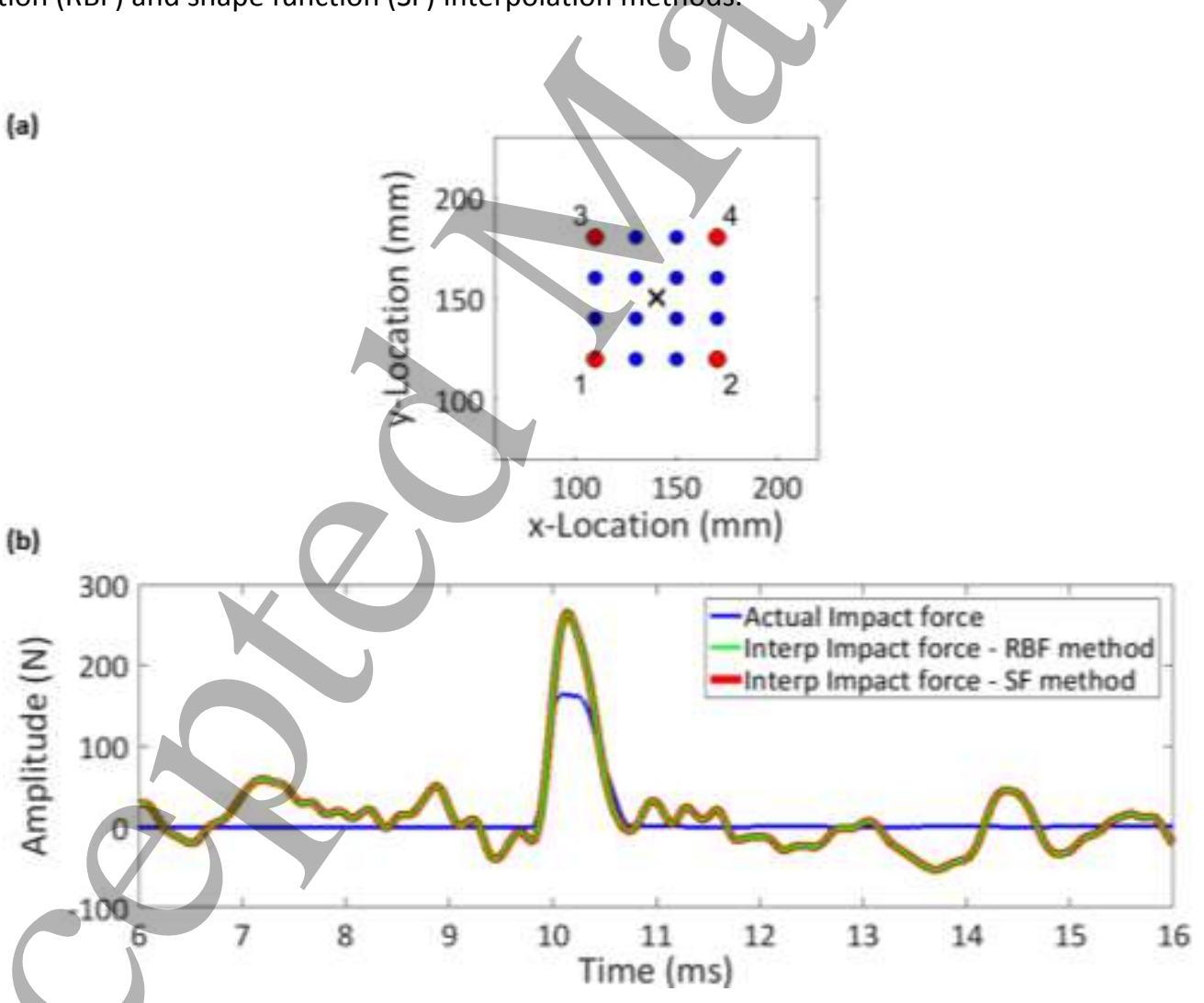

Figure 14. a) Zoom on the wing panel with a reduced section of the initial grid. The calculate impact location is shown as a cross $(X)$. b) Comparison between the actual impact force and the interpolated ones by using radial basis function (RBF) and shape function (SF) interpolation methods. 
Table 6. Comparison between impact forces considering set 2 of interpolation points on both specimens.

\begin{tabular}{cccccc}
\hline & & \multicolumn{3}{c}{ INTERPOLATED IMPACT FORCES } \\
\hline & & \multicolumn{2}{c}{ RBF method } & \multicolumn{2}{c}{ SF method } \\
& & CFRP plate & Wing panel & CFRP plate & Wing panel \\
\hline Error $\Gamma$ & $T=1 \mathrm{~ms}$ & 0.55 & 0.38 & 0.55 & 0.38 \\
& $T=3 \mathrm{~ms}$ & 1.04 & 0.73 & 1.04 & 0.73 \\
\hline
\end{tabular}

It should be noted that, as obtained by considering set 1 (see Table 4), the two interpolation algorithms generated the same results, as reported in Table 6. Comparing Table 4 and Table 6 it is evident that the error increases as the distance between the interpolation points is higher.

\subsection{Impact force reconstruction results - Set 3 of calibration points}

In some cases, the calibration process could not be performed on a dense initial grid, therefore information associated with points far from the impact location needed to be used with a high error in the interpolated results. However, unlike the SF method, the RBF interpolation technique is not limited to only four points (as in set 2, please see Sec. 5.3) and it was possible to take advantage of the information related to other calibration points far from impact event on a coarse initial grid. This is shown in Figure 15, Figure 16 and Table 7 where the information associated with four points closest to the impact on both specimens was not available. The SF interpolation method is applied considering the average between the results obtained by using the two closest to impact sets of calibration points (set 3), as depicted in Figure 15 and Figure 16. 
(a)

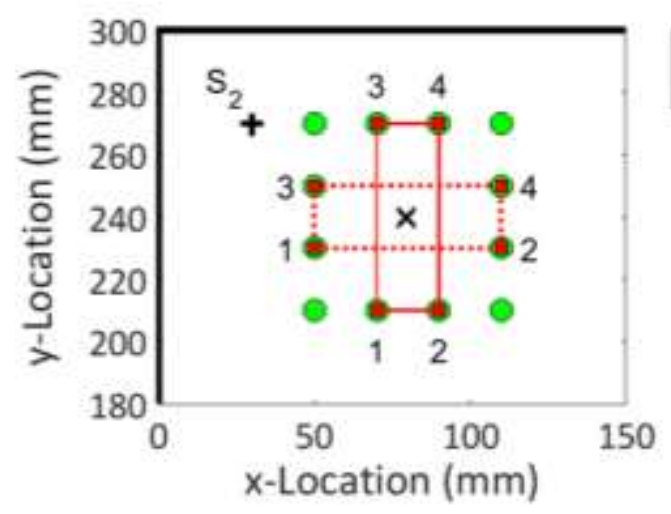

- RBF interpolation points
- SF interpolation points

(b)

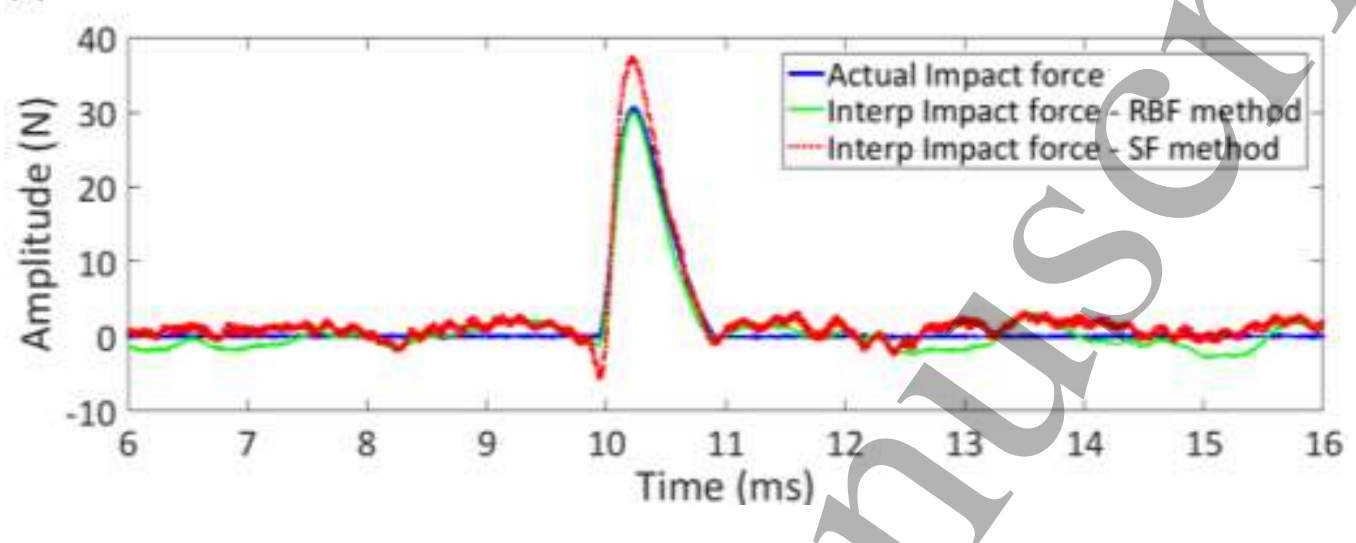

Figure 15. a) Zoom on the CFRP plate with the two sets 3 of interpolation points. The calculated impact location is shown as a cross $(x)$. b) Comparison between the actual impact force and the interpolated ones by using radial basis function (RBF) and shape function (SF) interpolation methods.

(a)

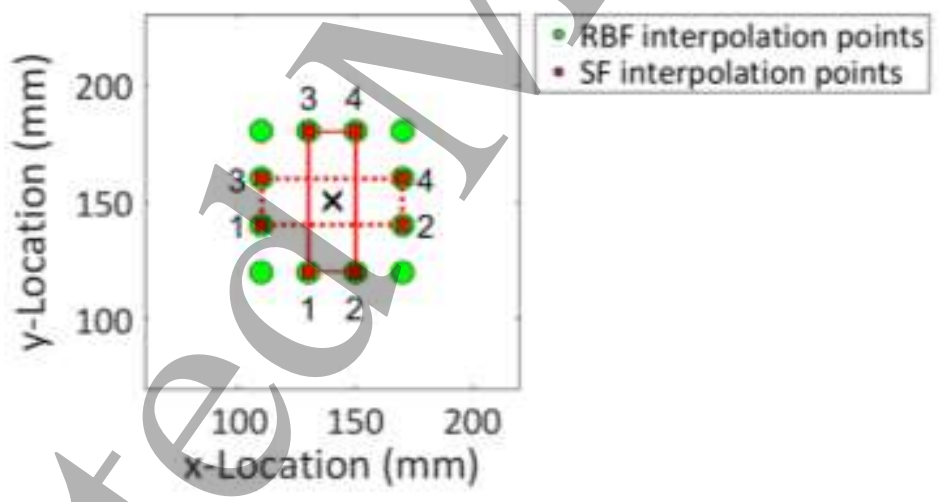

(b) $\quad x$-Location $(\mathrm{mm})$

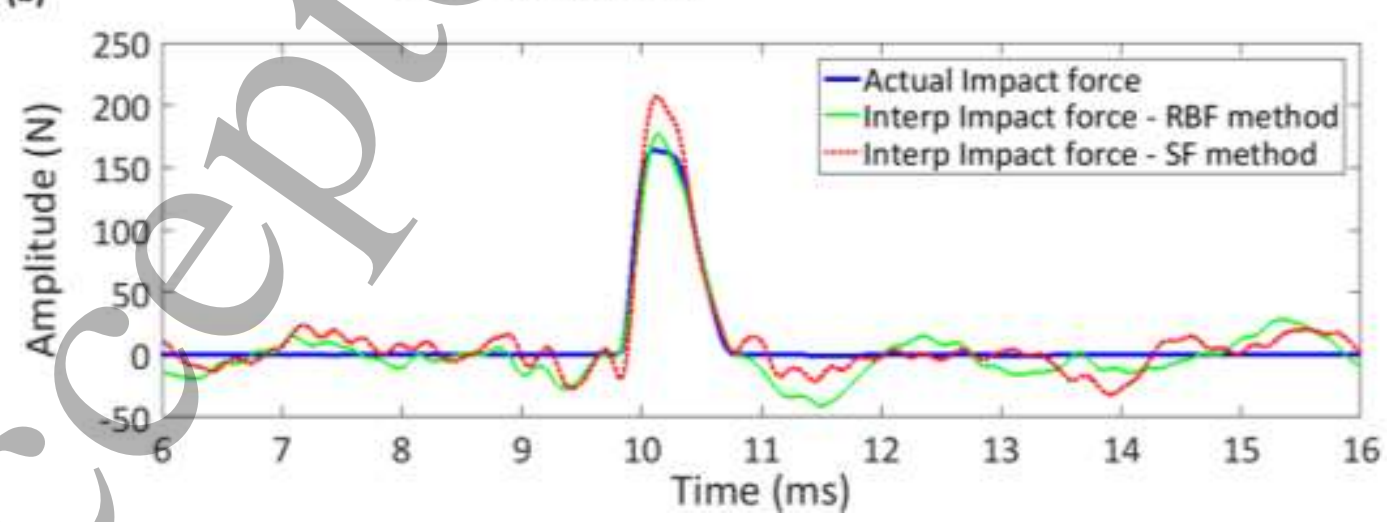

Figure 16. a) Zoom on the wing panel with the two sets 3 of interpolation points. The calculated impact location is shown as a cross $(X)$. b) Comparison between the actual impact force and the interpolated ones by using radial basis function (RBF) and shape function (SF) interpolation methods. 
Table 7. Comparison between impact forces considering the two sets 3 of interpolation points on both specimens.

\begin{tabular}{cccccc}
\hline & & \multicolumn{3}{c}{ INTERPOLATED IMPACT FORCES } \\
\hline & & \multicolumn{2}{c}{ RBF method } & \multicolumn{2}{c}{ SF method } \\
& & CFRP plate & Wing panel & CFRP plate & Wing panel \\
\hline Error $\Gamma$ & $T=1 \mathrm{~ms}$ & 0.09 & 0.07 & 0.17 & 0.18 \\
& $T=3 \mathrm{~ms}$ & 0.24 & 0.49 & 0.4 & 0.41 \\
\hline
\end{tabular}

As depicted in Figure 15 and Figure 16, the RBF interpolation method shows a good performance in detecting the maximum value of the impact force on both specimens ( $30 \mathrm{~N}$ and $150 \mathrm{~N})$, despite an higher error of the RBF method with respect to SF method if the wing panel is considered (see Table 7). This is due to a wider time window $(T=3 \mathrm{~ms})$. Nevertheless this result does not affect the validity of the approach in amplitude peak detection when RBF interpolation method is used.

As showed in the comparison between the CFRP plate and the wing panel, the validity of the described method is not dependent of the impact amplitude. Figure 17 and Table 8 illustrates the comparison results considering two different calibration processes on the CFRP plate, with maximum values of impact forces equal to $60 \mathrm{~N}$ and $90 \mathrm{~N}$. The'sets of calibration points are the same as used in Figure 15 and Figure 16 (set 3), where the information associated with four points closest to the impact on both specimens was not available. 


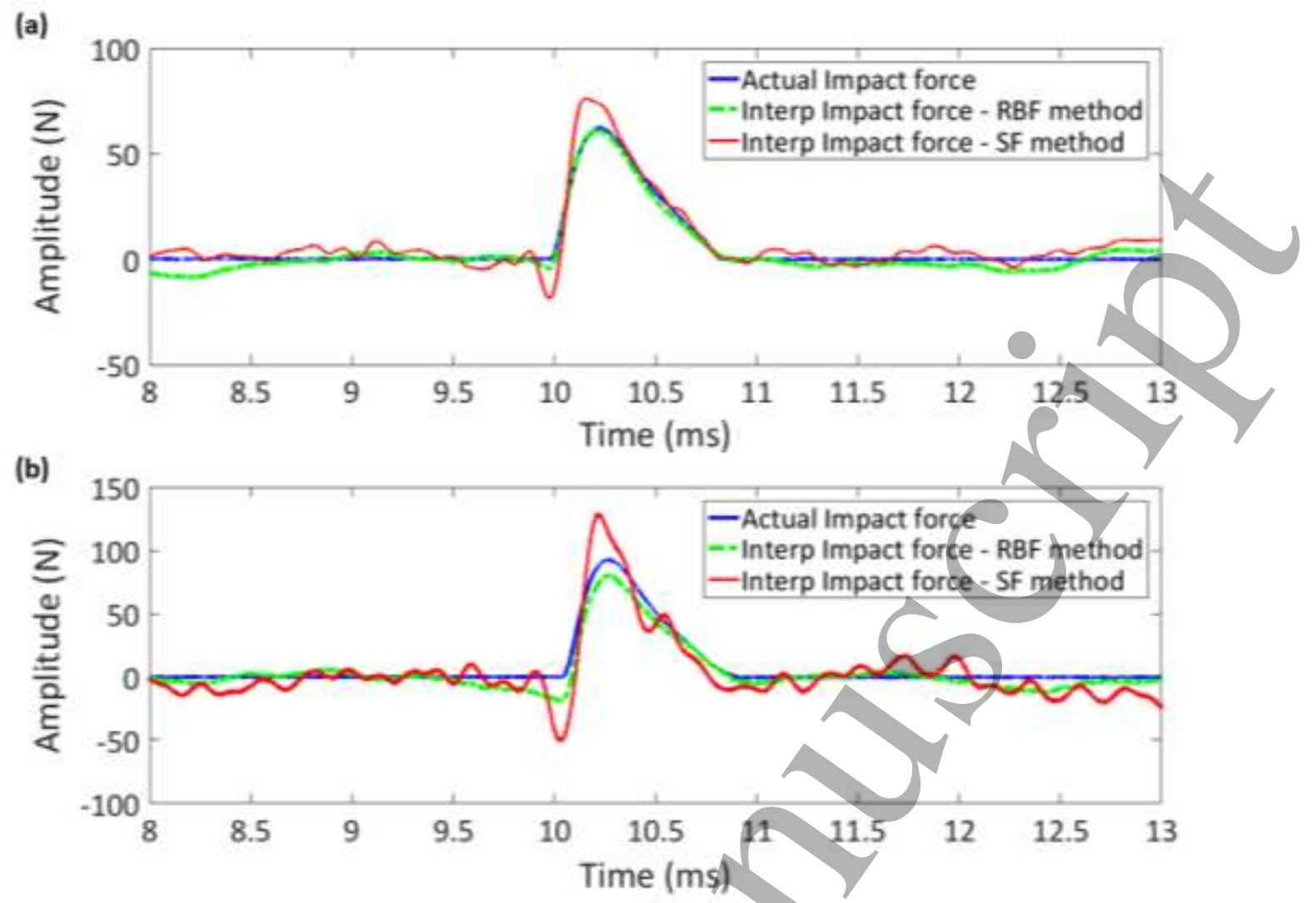

Figure 17. Comparison between the actual impact force and the interpolated ones on the CFRP plate by using radial basis function (RBF) and shape function (SF) interpolation methods, considering the set 3 of calibration points and impact peak amplitudes equal to: a) $60 \mathrm{~N}$ and b) $90 \mathrm{~N}$.

Table 8. Comparison between impact forces considering set 3 of interpolation points on the CFRP plate and different impact peak amplitudes.

\begin{tabular}{llll}
\hline & & \multicolumn{3}{c}{ INTERPOLATED IMPACT FORCES } \\
& & $\mathrm{RBF}$ & $\mathrm{SF}$ \\
\hline \multirow{2}{*}{$T=1 \mathrm{~ms}$} & Error $\Gamma(60 \mathrm{~N})$ & 0.06 & 0.18 \\
& Error $\Gamma(90 \mathrm{~N})$ & 0.24 & 0.44 \\
\hline \multirow{3}{*}{$T=3 \mathrm{~ms}$} & Error $\Gamma(60 \mathrm{~N})$ & 0.18 & 0.43 \\
& Error $\Gamma(90 \mathrm{~N})$ & 0.4 & 0.74 \\
\hline
\end{tabular}

The results confirmed the accuracy of the proposed method with respect to SF interpolation approach, with a maximum error less than $13 \mathrm{~N}$ in the detection of a $90 \mathrm{~N}$ impact peak (see Figure 17). In Figure 18 it is depicted the results comparison related to an impact event on a different location on the wing panel. The errors are reported in Table 9. 
(a)

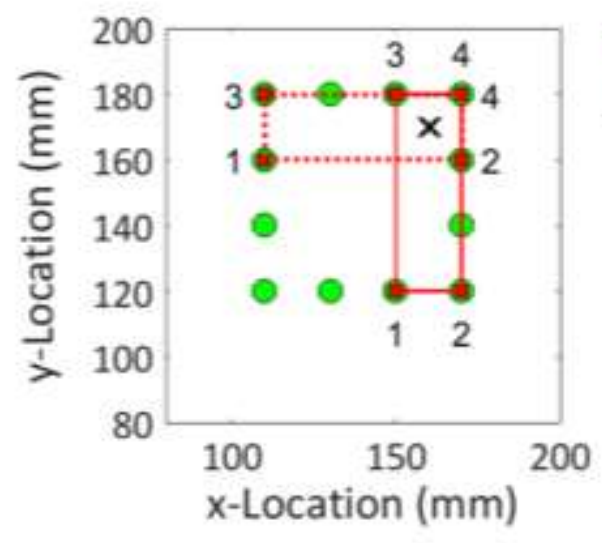

- RBF interpolation points

- SF interpolation points

(b)

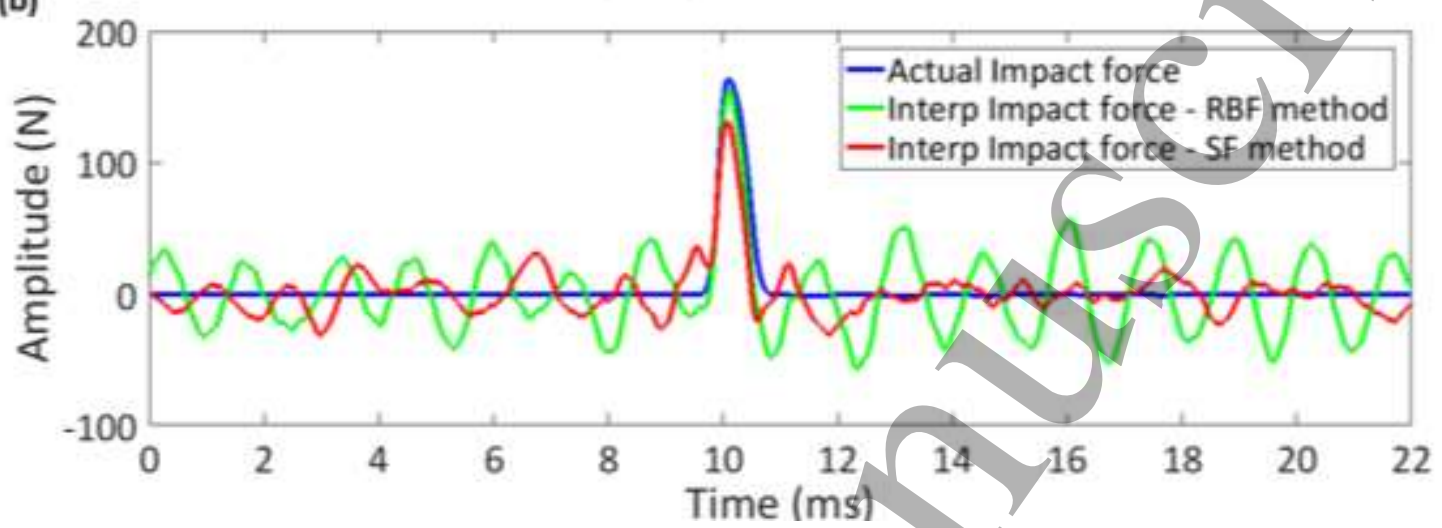

Figure 18. a) Zoom on the wing panel with the sets of interpolation points. The calculated impact location is shown as a cross $(X)$. b) Comparison between the actual impact force and the interpolated ones by using radial basis function (RBF) and shape function (SF) interpolation methods.

Table 9. Comparison between impact forces considering an impact at different location on the CFRP plate.

\begin{tabular}{llll}
\hline & & \multicolumn{2}{c}{ INTERPOLATED IMPACT FORCES } \\
\hline & & RBF & SF \\
\hline \multirow{2}{*}{ Error $\Gamma$} & $T=1 \mathrm{~ms}$ & 0.27 & 0.4 \\
& $T=3 \mathrm{~ms}$ & 0.62 & 0.79 \\
\hline
\end{tabular}

Despite the new impact location on the wing panel is not equally surrounded by the set point used in the RBF interpolation method (the green points in Figure 18), the obtained result is still better than the SF method (see Table 9).

It should be finally noted that, in spite of the hypothesis of linear relation between the impact force and the structural responses [Eqs. (2) and (11)], the obtained results on the real aeronautical structure (i.e. the wing panel) showed the validity of the proposed impact force reconstruction algorithm also 
in the presence of nonlinear effects due to the material and geometry. This confirmed the robustness of the presented approach.

\section{CONCLUSIONS}

This paper presented a hierarchical radial basis function algorithm for the reconstruction of the impact force. An initial calibration process was initially performed, which consists of acquiring and storing impact forces and structural responses from a set of excitation points on the specimen surface. The localisation of the impact event was achieved by using time reversal method, which was able to detect the impact coordinates with high level of accuracy. The reconstruction of the impact force was then obtained through an interpolation algorithm based on hierarchical radial basis functions. Such a reconstruction algorithm involved the interpolation of transfer functions calculated during the calibration process by using a Fourier method able to preserve the magnitudes and phases of measured signals. A number of experimental impact tests were performed on a CFRP composite plate-like structure and an aluminium/CFRP composite wing-stringer-skin panel in order to validate the proposed methodology on full-scale aircraft structures. The radial basis function interpolation method provided an accurate reconstruction of the impact force with a perfect matching of the impact peak. Moreover, this algorithm was compared with an interpolation approach available in literature, which was based on polynomial shape functions. Impact force reconstruction results revealed that the proposed hierarchal algorithm provided higher accuracy, especially when calibration points far from the impact location were considered. The proposed impact location and force reconstruction approach were applied on a real aeronautical structure since no information about the material and geometry was needed, thus allowing for faster and timely inspections.

\section{REFERENCES}

1. Kundu, T., Nakatani, H., \& Takeda, N. (2012). Acoustic source localization in anisotropic plates. Ultrasonics, 52(6), 740-746. 
2. Meo, M., Zumpano, G., Piggott, M., \& Marengo, G. (2005). Impact identification on a sandwich plate from wave propagation responses. Composite structures, 71(3), 302-306.

3. Tobias, A. (1976). Acoustic-emission source location in two dimensions by an array of three sensors. Nondestructive testing, 9(1), 9-12.

4. Jacquelin, E., Bennani, A., \& Hamelin, P. (2003). Force reconstruction: analysis and regularization of a deconvolution problem. Journal of Sound and Vibration, 265(1), 81-107.

5. Doyle, J. F. (1987). Determining the contact force during the transverse impact of plates. Experimental Mechanics, 27(1), 68-72.

6. Doyle, J. F. (1987). Experimentally determining the contact force during the transverse impact of an orthotropic plate. Journal of Sound and Vibration, 118(3), 441-448.

7. Chang, C., \& Sun, C. T. (1989). Determining transverse impact force on a composite laminate by signal deconvolution. Experimental mechanics, 29(4), 414-419.

8. Wu, E., Tsai, T. D., \& Yen, C. S. (1995). Two methods for determining impact-force history on elastic plates. Experimental mechanics, 35(1), 11-18.

9. Wu, E., Yeh, J. C., \& Yen, C. S. (1994). Identification of impact forces at multiple locations on laminated plates. AIAA journal, 32(12).

10. Kalhori, H., Ye, L., \& Mustapha, S. (2017). Inverse estimation of impact force on a composite panel using a single piezoelectric sensor. Journal of Intelligent Material Systems and Structures, 28(6), 799-810.

11. Kalhori, H., Alamdari, M. M., \& Ye, L. (2018). Automáted algorithm for impact force identification using cosine similarity searching. Measurement, 122, 648-657.

12. Qiao, B., Zhang, X., Gao, J., \& Chen, X. (2016). Impact-force sparse reconstruction from highly incomplete and inaccurate measurements. Journal of Sound and Vibration, 376, 72-94.

13. Qiao, B., Zhang, X., Gao, J., Liu, R., \& Chen, X. (2017). Sparse deconvolution for the large-scale ill-posed inverse problem of impact force reconstruction. Mechanical Systems and Signal Processing, 83, 93-115.

14. Pan, C. D., Yu, L., Liu, H. L., Chen, Z. P., \& Luo, W. F. (2018). Moving force identification based on redundant concatenated dictionary and weighted 11-norm regularization. Mechanical Systems and Signal Processing, 98, 3249.

15. Yan, G., Sun, H., \& Büyüköztürk, O. (2017). Impact load identification for composite structures using Bayesian regularization and unscented Kalman filter. Structural Control and Health Monitoring, 24(5), e1910.

16. Park, J., Ha, S., \& Chang, F. K. (2009). Monitoring impact events using a system-identification method. AIAA $J, 47(9), 2011-2021$. 
17. Chen, C., \& Yuan, F. G. (2010). Impact source identification in finite isotropic plates using a time-reversal method: theoretical study. Smart Materials and Structures, 19(10), 105028.

18. Chen, C., Li, Y., \& Yuan, F. G. (2012). Impact source identification in finite isotropic plates using a time-reversal method: experimental study. Smart Materials and Structures, 21(10), 105025.

19. Xu, L., Wang, Y., Cai, Y., Wu, Z., \& Peng, W. (2016). Determination of impact events on a plate-like composite structure. The Aeronautical Journal, 120(1228), 984-1004.

20. Jones, R. T., Sirkis, J. S., \& Friebele, E. J. (1997). Detection of impact location and magnitude for isotropic plates using neural networks. Journal of intelligent material systems and structures, 8(1), 90-99.

21. Worden, K., \& Staszewski, W. J. (2000). Impact location and quantification on a composite panel using neural networks and a genetic algorithm. Strain, 36(2), 61-68.

22. Haywood, J., Coverley, P. T., Staszewski, W. J., \& Worden, K. (2004). An automatic impact monitor for a composite panel employing smart sensor technology. Smart Materials and Structures, 14(1), 265.

23. Ghajari, M., Sharif-Khodaei, Z., Aliabadi, M. H., \& Apicella, A. (2013). Identification of impact force for smart composite stiffened panels. Smart Materials and Structures, 22(8), 085014.

24. Sarego, G., Cappellini, L., Zaccariotto, M., \& Galvanetto, U. (2017). Impact force reconstruction in composite panels. Procedia Structural Integrity, 5, 107-114.

25. Ciampa, F., \& Meo, M. (2012). Impact detection in anisotropic materials using a time reversal approach. Structural Health Monitoring, 11(1), 43-49.

26. Ciampa, F., \& M. Meo. (2014). Impact localization on a composite tail rotor blade using an inverse filtering approach. Journal of Intelligent Material Systems and Structures, 25(15), 1950-1958.

27. Ciampa, F., Boccardi S., \& M. Meo. (2016). Factors affecting the imaging of the impact location with inverse filtering and diffuse wave fields. Journal of Intelligent Material Systems and Structures, 27(11), 1523-1533.

28. Ing, R. K., Quieffin, N., Catheline, S., \& Fink, M. (2005). In solid localization of finger impacts using acoustic time-reversal process. Applied Physics Letters, 87(20), 204104.

29. Ciampa, F., \& Meo, M. (2010). Acoustic emission source localization and velocity determination of the fundamental mode $\mathrm{A}_{0}$ using wayelet analysis and a Newton-based optimization technique. Smart Materials and Structures, 19(4), 045027.

30. Ciampa, F., \& Meo, M. (2010). A new algorithm for acoustic emission localization and flexural group velocity determination in anisotropic structures. Composites Part A: Applied Science and Manufacturing, 41(12), 17771786.

31. Ciampâ, F., Meo, M., \& Barbieri, E. (2012). Impact localization in composite structures of arbitrary cross 
section. Structural Health Monitoring, 11(6), 643-655.

32. De Simone, M. E., Ciampa, F., Boccardi, S., \& Meo, M. (2017). Impact source localisation in aerospace composite structures. Smart Materials and Structures, 26(12), 125026.

33. Aldaz, J. M., Barza, S., Fujii, M., \& Moslehian, M. S. (2015). Advances in Operator Cauchy-Schwarz inequalities and their reverses. Annals of Functional Analysis, 6(3), 275-295.

34. Katznelson, Y. (2004). An introduction to harmonic analysis. Cambridge University Press.

35. Thiene, M., Ghajari, M., Galvanetto, U., \& Aliabadi, M. H. (2014). Effects of the transfer function evaluation on the impact force reconstruction with application to composite panels. Composite Structures, 114, 1-9.

36. Haar, A. (1918). Die minkowskische geometrie und die ann an stetige funktionen. Math. Ann, 18, $294-311$.

37. Cheney, E. W. (1966). Introduction to approximation theory McGraw-Hill Boøk Co. New York.

38. Hardy, R. L. (1971). Multiquadric equations of topography and other irregular surfaces. Journal of geophysical research, 76(8), 1905-1915.

39. Davis, P. J. (1975). Interpolation and approximation. Dover, New York.

40. Powell, M. J. D. (1981). Approximation theory and methods. Cambridge University Press.

41. Rippa, S. (1984). Interpolation and smoothing of scattered data by radial basis functions. Tel Aviv University. Master's thesis.

42. Micchelli, C. A. (1986). Interpolation of scattered data: distance matrices and conditionally positive definite functions. Constructive approximation, 2(1), 11-22.

43. Bookstein, F. L. (1989). Principal warps: Thin-plate splines and the decomposition of deformations. IEEE Transactions on pattern analysis and machine intelligence, 11(6), 567-585.

44. Carlson, R. E., \& Foley, T. A. (1991). The parameter R2 in multiquadric interpolation. Computers \& Mathematics with Applications, 21(9), 29-42.

45. Foley, T. A. (1994). Near optimal parameter selection for multiquadric interpolation. J. Appl. Sci. Comput, 1, 5469.

46. Fornberg, B. (1998). A practical guide to pseudospectral methods (Vol. 1). Cambridge University Press.

47. Freeden, W., Geryens, T., \& Schreiner, M. (1998). Constructive approximation on the sphere with applications to geomathematics. Oxford University Press.

48. Cucker, F., \& Smale, S. (2002). On the mathematical foundations of learning. Bulletin of the American mathematical society, 39(1), 1-49.

49. Wright, G. B. (2003). Radial basis function interpolation: numerical and analytical developments. University of Colorado, Boulder. Doctoral dissertation. 
50. Fornberg, B., \& Flyer, N. (2005). Accuracy of radial basis function interpolation and derivative approximations on 1-D infinite grids. Advances in Computational Mathematics, 23(1), 5-20.

51. Schaback, R., \& Wendland, H. (2006). Kernel techniques: from machine learning to meshless methods. Acta numerica, 15, 543-639.

52. Ferreira, A. J., Kansa, E. J., Fasshauer, G. E., \& Leitão, V. M. A. (Eds.). (2009). Progress on meshless methods. Berlin, Germany: Springer.

53. Elder, J. (2009). Handbook of statistical analysis and data mining applications. Academic Press.

54. Skala, V. (2016). A practical use of radial basis functions interpolation and approximation. Proceedings of revista investigacion operacional, 37(2), 137-145.

55. Ciampa, F., Pickering, S. G., Scarselli, G., \& Meo, M. (2017). Nonlinear imaging of damage in composite structures using sparse ultrasonic sensor arrays. Structural Control and Health Monitoring, 24(5).

56. Atkinson, K. E. (2008). An introduction to numerical analysis. John Wiley \& Sons.

57. Paget, C. A., Atherton, K., \& O’Brien, K. (2003). Triangulation algorithm for damage location in aeronautical composite structures. Proc. $4^{\text {th }}$ Int. Workshop on Structural Health Monitoring (Stanford, CA), 363-370. 